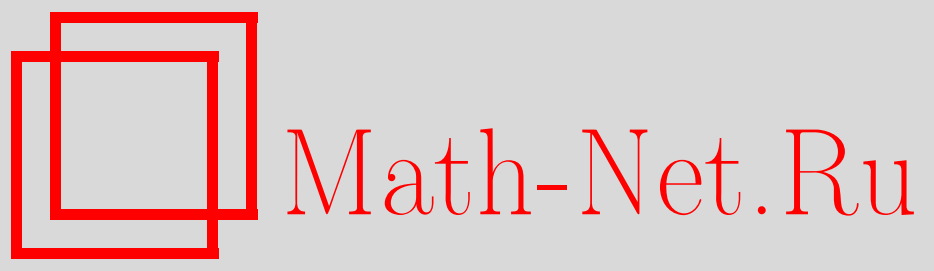

Д. С. Романов, Метод синтеза легкотестируемых схем, допускающих единичные проверяющие тесты константной длины, Дискрет. матем., 2014, том 26, выпуск 2, 100-130

DOI: https://doi.org/10.4213/dm1283

Использование Общероссийского математического портала Math-Net.Ru подразумевает, что вы прочитали и согласны с пользовательским соглашением http: //www . mathnet.ru/rus/agreement

Параметры загрузки:

IP : 3.85 .5 .30

26 апреля 2023 г., 13:33:34

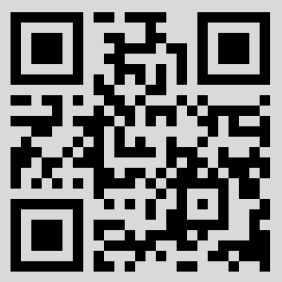




\title{
Метод синтеза легкотестируемых схем, допускающих единичные проверяющие тесты константной длины
}

(๑) 2014 г. Д. С. Романов*

\begin{abstract}
Предлагается метод синтеза неизбыточных схем из функциональных элементов в произвольном полном базисе, реализующих произвольные отличные от констант булевы функции и допускающих единичные проверяющие тесты длины не более 4 при инверсных и произвольных константных неисправностях на выходах элементов.
\end{abstract}

Ключевые слова: схема из функциональных элементов, единичный проверяющий тест, константная неисправность на выходе элемента, константная неисправность на выходе элемента, функция Шеннона длины теста, легкотестируемая схема.

\section{Введение и основные определения}

Схемы из функциональных элементов (СФЭ) или комбинационные схемы - одна из классических моделей управляющих систем без памяти. Анализ функционирования СФЭ при возникновении в них неисправностей традиционно осуществляется с помощью тестового подхода, предложенного в работах С. В. Яблонского и И. А. Чегис $[1,2]$ в середине $1950-$ х годов. В рамках этого подхода предполагается, что на схему действует источник неисправностей, способный преобразовать эту схему к одной из схем некоторого заранее известного списка (конечной длины), содержащего и исходную схему. Действие источника неисправностей однократное, то есть во время исследования схемы источник неисправностей уже перестал действовать на схему. Исследование схемы заключается в подаче на входы схемы входных наборов и в изучении выходных значений на этих наборах. При этом формулируется цель контроля - некоторое множество пар функционирований схем из упомянутого конечного списка. Под тестом для заданных схемы и цели контроля понимается множество входных наборов, на котором любая пара функционирований (при условии их неравенства) из цели контроля отличима на наборах теста.

Пусть $f\left(\tilde{x}^{n}\right)$ - произвольная булева функция, зависящая (существенно или фиктивно) от переменных $x_{1}, x_{2}, \ldots, x_{n}$, а $S$ - схема из функциональных элементов в некотором базисе $B$, реализующая функцию $f$ (все определения, не введенные в данной работе, можно найти в монографии [3]). Пусть на схему $S$ действует

*Место работы: МГУ им. М. В. Ломоносова, e-mail: romanov@cs.msu.ru

Работа выполнена при поддержке РФФИ (проекты № 12-01-00964-а и № 13-01-00958-а). 
источник одиночных неисправностей $U$, вызывающий инверсные или константные неисправности на выходах функциональных элементов, то есть на выходе любого (не более чем одного) функционального элемента схемы вместо реализуемой на его выходе функции от его входов может реализовываться либо отрицание этой функции (случай инверсной неисправности), либо произвольная булева константа (случай константной неисправности). Нетривиальной будем называть такую неисправность схемы $S$, в результате которой значение на выходе хотя бы одного функционального элемента $E$ (схемы $S$ ) при хотя бы одном входном наборе $\tilde{\alpha}^{n}$ не равно значению на выходе элемента $E$ на наборе $\tilde{\alpha}^{n}$ при отсутствии неисправностей в схеме $S$. Обозначим через $W(S)$ множество всех попарно не равных функций, каждая из которых может быть реализована схемой, полученной из схемы $S$ в результате действия источника неисправностей $U$ (в частности, $f \in W(S)$ ). Схема $S$ называется неизбыточной тогда и только тогда, когда для любой нетривиальной неисправности (вызванной действием на схему $S$ источника неисправностей $U$ ) для функции $g\left(\tilde{x}^{n}\right)$, реализуемой той неисправной схемой, которая получена в результате действия данной неисправности, имеет место неравенство: $f\left(\tilde{x}^{n}\right) \neq g\left(\tilde{x}^{n}\right)$.

Множество $T$ наборов значений переменных $x_{1}, x_{2}, \ldots, x_{n}$ называется единичным проверяющим тестом для схемы $S$ относительно инверсных и константных неисправностей на выходах элементов тогда и только тогда, когда для любой функции $g$ из $W(S)$ такой, что $g\left(\tilde{x}^{n}\right) \neq f\left(\tilde{x}^{n}\right)$, найдется набор $\tilde{\alpha}$ из $T$, для которого выполнено неравенство $f(\tilde{\alpha}) \neq g(\tilde{\alpha})$. (Понятие единичного проверяющего теста относительно константных неисправностей на выходах элементов вводится аналогичным образом.) Количество различных наборов в тесте $T$ называется его длиной и обозначается через $l(T)$ или через $|T|$. Тест минимальной длины называется минималъным. Обозначим через $D(S)$ длину минимального единичного проверяющего теста относительно инверсных и константных неисправностей на выходах элементов в схеме $S$, через $D_{B}\left(f\left(\tilde{x}^{n}\right)\right)$ - минимум величины $D(S)$ по всем неизбыточным реализующим $f\left(\tilde{x}^{n}\right)$ схемам $S$ в базисе $B$. При этом будем считать, что если для какой-то функции $f$ не существует неизбыточных схем, ее реализующих, то $D_{B}(f)=0$. Пусть $\hat{P}_{2}^{n}$ - множество всех булевых функций, существенно зависящих от всех своих переменных $x_{1}, x_{2}, \ldots, x_{n}$ (в частности, $\hat{P}_{2}^{0}=\{0,1\}$ ). Всюду в дальнейшем будем, не ограничивая общности, считать, что любая не равная тождественно константе функция $f\left(\tilde{x}^{n}\right)$ существенно зависит от всех своих переменных. Через $D_{B}(n)$ обозначим функцию Шеннона длинь единичного проверяющего теста относительно инверсных и константных неисправностей на выходах элементов, т. е. функцию

$$
D_{B}(n)=\max _{f\left(\tilde{x}^{n}\right) \in \hat{P}_{2}^{n}} D_{B}\left(f\left(\tilde{x}^{n}\right)\right) .
$$

Отметим, что задача отыскания верхних оценок функции Шеннона длины проверяющего теста для СФЭ может считаться корректной (в приведенных постановках), если неравенство $D_{B}(f)>0$ выполнено для доли функций $f$ из $\hat{P}_{2}^{n}$, стремящейся к единице с ростом $n$ к бесконечности.

В работе С. М. Редди [4] было доказано, что в базисе Жегалкина $\{x \& y, x \oplus y, 1\}$ функция Шеннона длины единичного проверяющего теста относительно произвольных константных неисправностей на выходах и входах элементов не превосходит $n+4$. Н. П. Редькиным было установлено, что в стандартном базисе $B_{0}=\{x \& y, x \vee y, \bar{x}\}$ функция Шеннона длины полного проверяющего теста относительно однотипных константных неисправностей на выходах элементов не превосходит $n$ [5], а функция Шеннона длины полного проверяющего теста относительно 
произвольных константных неисправностей на выходах элементов при всех натуральных $n$ не превосходит $O\left(2^{n / 2}\right)$ [6]. С. В. Коваценко [7] установил, что в случае $B=\{x \& y, x \oplus y, 1\}$ функция Шеннона длины единичного проверяющего теста относительно инверсных неисправностей на выходах элементов равна 1. Н. П. Редькин [8] доказал, что в произвольном полном базисе функция Шеннона длины единичного проверяющего теста относительно инверсных неисправностей на выходах элементов не превосходит 3. Ю. В. Бородина установила [9], что в стандартном базисе $B_{0}$ функция Шеннона длины полного проверяющего теста относительно однотипных константных неисправностей на выходах элементов равна 2. Ею же доказано [10], что функция Шеннона длины единичного проверяющего теста относительно константных неисправностей типа 1 на выходах элементов в базисе Жегалкина равна 1. Ю. В. Бородина и П. А. Бородин получили [11], что функция Шеннона длины полного проверяющего теста относительно константных неисправностей типа 0 на выходах элементов в базисе Жегалкина равна 1. С. С. Коляда [12-14] доказал, что для любого $n \in \mathbb{N}$ в произвольном полном базисе функция Шеннона длины единичного проверяющего теста относительно произвольных константных неисправностей на выходах элементов не превосходит $n+3$. Автором настоящей статьи найдены два таких примера полных базисов функциональных элементов $\check{B}^{\prime}, \check{B}^{\prime \prime}$, что $D_{\check{B}^{\prime}}(n) \leqslant 4$ [15], а в базисе $\check{B}^{\prime \prime}$ функция Шеннона длины полного проверяющего теста относительно произвольных константных неисправностей на выходах элементов не превосходит 4 [16] при любых целых неотрицательных $n$. Отметим, что В. Н. Носков установил для каждой булевой функции $f\left(\tilde{x}^{n}\right)$ существование в произвольном полном базисе такой схемы $S$ с тремя дополнительными входами и одним дополнительным выходом, что функция $f$ является подфункцией одной из двух функций, реализуемой схемой $S$, а длина теста, проверяющего не более чем $m$ произвольных неисправностей элементов или блоков схемы, ведет себя как $O\left(n+2^{m}\right)$ [17].

В данной работе устанавливается, что длина минимального единичного проверяющего теста относительно инверсных и произвольных константных неисправностей на выходах элементов в произвольном полном базисе для каждой неконстантной булевой функции лежит в пределах от 2 до 4. При этом сначала данный факт будет установлен для базиса $B^{\prime}=\{x \& y, x \oplus y, x \sim y\}$, а затем переход к произвольному базису с использованием техники расширений базисов будет осуществлен на методологических основах работ Н. П. Редькина $[6,8]$ и под существенным влиянием работы [8].

\section{Вспомогательные леммы}

Две СФЭ $\Sigma^{\prime}$ и $\Sigma^{\prime \prime}$ называются эквивалентными тогда и только тогда, когда у них одинаковы множества входов, одинаковы множества выходов (с учетом названий, сопоставленных входам и выходам переменных) и при этом данные СФЭ реализуют равные системы функций (то есть функции, реализуемые в выходных вершинах этих схем с одинаковыми выходными переменными, равны).

Пусть СФЭ $\Sigma^{\prime}$ является подсхемой схемы $\Sigma$. Тогда выполнены следующие импликации. Если вершина $v$ схемы $\Sigma^{\prime}$ является входом (выходом) в схеме $\Sigma$, то она является входом (соответственно, выходом) в подсхеме $\Sigma^{\prime}$. Если вершина $v$ схемы $\Sigma$ является истоком в схеме $\Sigma^{\prime}$ и не является входом схемы $\Sigma$, то она объявляется входом в подсхеме $\Sigma^{\prime}$ (и ей приписывается новый символ входной переменной). Если из вершины $v$ подсхемы $\Sigma^{\prime}$ исходит хотя бы одна дуга в вершины вне подсхемы 
$\Sigma^{\prime}$, то вершина $v$ объявляется выходом в подсхеме $\Sigma^{\prime}$ (и ей приписывается новый символ выходной переменной). Любая вершина подсхемы $\Sigma^{\prime}$ может быть объявлена в ней выходом.

Пусть теперь СФЭ $\Sigma^{\prime}$ является подсхемой схемы $\Sigma$, и при этом схемы $\Sigma^{\prime}$ и $\Sigma^{\prime \prime}$ эквивалентны. Такая замена подсхемы $\Sigma^{\prime}$ на подсхему $\Sigma^{\prime \prime}$, при которой входы (выходы) схемы $\Sigma^{\prime}$ заменяются на одноименные входы (выходы) схемы $\Sigma^{\prime \prime}$, называется эквивалентной подстановкой. При эквивалентной подстановке схема $\Sigma$ преобразуется к эквивалентной ей схеме.

Лемма 1. Если некоторое множество наборов $T$ образует единичный проверяющий тест относительно произвольных константных неисправностей на выходах элементов в неизбыточной схеме $S$, то это же множество наборов $T$ образует единичный проверяющий тест относительно инверсных и произвольных константных неисправностей на выходах элементов в схеме $S$.

Доказательство следует из того, что инверсная неисправность на выходе фиксированного элемента обнаруживается на всяком входном наборе, на котором обнаруживается хоть какая-то константная неисправность на выходе этого элемента.

Замечание 1. В дальнейшем в силу леммы 1 одиночные инверсные неисправности не будут рассматриваться отдельно, и верхние оценки длин проверяющих тестов относительно одиночных инверсных и произвольных константных неисправностей на выходах элементов будут получаться автоматически из аналогичных результатов для одиночных произвольных константных неисправностей на выходах элементов.

Лемма 2. Пусть множество Т входных наборов неизбыточной схемы $S$ образует единичный проверяющий тест относительно произвольных константных неисправностей на выходах элементов в схеме $S, A \subseteq T$, на каждом наборе из $A$ обнаруживается какая-то константная неисправность на выходе элемента $E$ схемъ $S$, и псть $J$ - множество всех наборов значений на входах элемента $E$, появляющихся при подаче на входы схемы $S$ всех наборов из А. Пусть неизбыточная схема $\Sigma_{E}$ при отсутствии неисправностей эквивалентна элементу $E$ (то есть реализует ту же функиию), и $J$ - единичный проверяющий тест относительно произвольных константных неисправностей на выходах элементов в схеме $\Sigma_{E}$. Тогда схема $S^{\prime}$, полученная эквивалентной подстановкой вместо элемента $E$ подсхемы $\Sigma_{E}$, неизбыточна, и множество наборов $T$ образует единичный проверяющий тест относительно произвольных константных неисправностей на выходах элементов в схеме $S^{\prime}$.

Доказательство. Очевидно, требуется доказать, что любая одиночная неисправность $H^{\prime}$ в схеме $\Sigma_{E}$ как подсхеме схемы $S^{\prime}$ будет обнаружена на множестве $T$. Пусть эта неисправность обнаруживается в схеме $\Sigma_{E}$ на наборе $\beta$ из $J$. Тогда найдется набор $\alpha$ из $A$, порождающий на входах $\Sigma_{E}$ в $S^{\prime}$ набор $\beta$. Так как на наборе $\alpha$ обнаруживается какая-то константная неисправность $H$ на выходе элемента $E$ схемы $S$, то значения (при неисправности $H$ ) на этом наборе как на выходе $E$ в $S$, так и на выходе схемы $S$, отличаются от соответствующих значений при отсутствии неисправностей в схеме $S$. Но, поскольку неисправность $H^{\prime}$ в $\Sigma_{E}$ обнаруживается на наборе $\beta$, значение на выходе $\Sigma_{E}$ при неисправности $H^{\prime}$ отличается от правильного и совпадает со значением на выходе $E$ в $S$ при неисправности $H$. Значит, неисправность $H^{\prime}$ в $\Sigma_{E}$ как подсхеме схемы $S^{\prime}$ обнаруживается на наборе $\alpha$, что и требовалось доказать. 
Лемма 3. Пусть схема $S^{\prime}$ получена из $S$ эквивалентной заменой элемента Е элементом $E$ с подсоединенной $\kappa$ его выходу цепочкой из четного числа инверторов (при этом, если выход Е ветвился, то ветвления переносятся на выход иепочки). Тогда, если одна из схем $S^{\prime}, S$ неизбыточна, то другая тоже неизбыточна, $u$ множества их единичных проверяющих тестов относительно произвольных константных неисправностей на выходах элементов совпадают.

Лемма 4. Пусть схема $S^{\prime}$ получена из $S$ следующим образом: вход схемы $x_{i}$ переносится в новую вершину и соединяется с бывшей входной вершиной $x_{i}$ через добавленный инвертор (при этом, если вход схемы $x_{i}$ ветвился, то ветвления переносятся на выход добавленного инвертора). Тогда, если схема $S^{\prime}$ неизбыточна, то $S$ тоже неизбыточна, и множества их единичных проверяющих тестов относительно произвольных константных неисправностей на выходах элементов получаются одно из другого инвертированием значений переменной $x_{i}$.

Лемма 5. Пусть схема $S^{\prime}$ получена из $S$ присодинением инвертора к выходу схемы с переносом этого выхода на выход добавленного инвертора. Тогда, если одна из схем $S^{\prime}, S$ неизбыточна, то другая тоже неизбыточна, и множества их единичных проверяющих тестов относительно произвольных константных неисправностей на выходах элементов совпадают.

Доказательство лемм 3-5 очевидны.

Лемма 6. Для произвольного полного базиса $B$ при всех $n \in \mathbb{N}$ имеет место неравенство $D_{B}(n) \geqslant 2$.

Доказательство следует из того, что каждую не равную константе функцию $f\left(\tilde{x}^{n}\right)$ необходимо отличать от обеих констант.

Лемма 7. Пусть выход функционального элемента $E$ в СФЭ $\Sigma$ не ветвится $u$ от выхода этого функционального элемента $\kappa$ выходу схемы $\Sigma$ ведет иепочка $Z$, не содержащая функциональных элементов, отличных от элементов суммы по модулю 2, эквивалентности и отрицания. Если на множестве А входных наборов схемы $\Sigma$ на выходе функиионального элемента $E$ возникают оба булевых значения, то на множестве А обнаруживаются обе константные неисправности на выходе элемента $E$ (при условии отсутствия иных неисправностей в схеме $\Sigma$ ), и на каждом наборе из А обнаруживается какая-то константная неисправность на выходе элемента $E$.

Доказательство. Пусть значение $\gamma$ на выходе функционального элемента $E$ возникает на входном наборе $\tilde{\alpha}$ из $A$. Поскольку при неисправности типа $\bar{\gamma}$ на выходе $E$ (при условии отсутствия иных неисправностей в схеме $\Sigma$ ) на входы всех элементов цепочки $Z$, кроме одного (на который подается выход $E$ ), подаются такие же значения, как при работе схемы в отсутствие неисправностей, а на этот единственный вход подается „неправильное“ значение, заметим: на выходе цепочки $Z$ (т. е. на выходе схемы $\Sigma$ ) возникнет отрицание того значения, которое возникало бы на наборе $\tilde{\alpha}$ в отсутствие неисправностей, и, значит, на наборе $\tilde{\alpha}$ обнаруживается константная неисправность $\bar{\gamma}$ на выходе функционального элемента $E$, что и требовалось доказать.

Введем обозначения: $\psi(x, y, z)=\bar{x}(y \vee z) \vee x(y \sim z), \psi^{\prime}(x, y, z)=\psi(x, y, z) \oplus x \bar{y} z$, столбцы значений этих функций имеют, соответственно, вид: (01111001), (01111101). 
Обозначим через $B^{\prime}, B^{\prime \prime}$ и $B_{1}$ следующие (функционально полные) базисы из функциональных элементов: $B^{\prime}=\{x \& y, x \oplus y, x \sim y\}, B^{\prime \prime}=\{\bar{x}, x \& \bar{y}, x \oplus y, x \sim y\}$, $B_{1}=\{\bar{x}, x \& y\}$.

Определим схемы $\hat{S}_{B^{\prime}}^{\psi}$ и $\hat{S}_{B^{\prime}}^{\psi^{\prime}}$, реализующие функции $\psi(x, y, z)$ и $\psi^{\prime}(x, y, z)$ в базисе $B^{\prime}$, в соответствии с рисунками 1 и 2 (соответственно), а также схемы $\hat{S}_{B^{\prime \prime}}^{\psi}$ и $\hat{S}_{B^{\prime \prime}}^{\psi^{\prime}}$, реализующие функции $\psi(x, y, z)$ и $\psi^{\prime}(x, y, z)$ в базисе $B^{\prime \prime}$, в соответствии с рисунками 3 и 4 (соответственно).

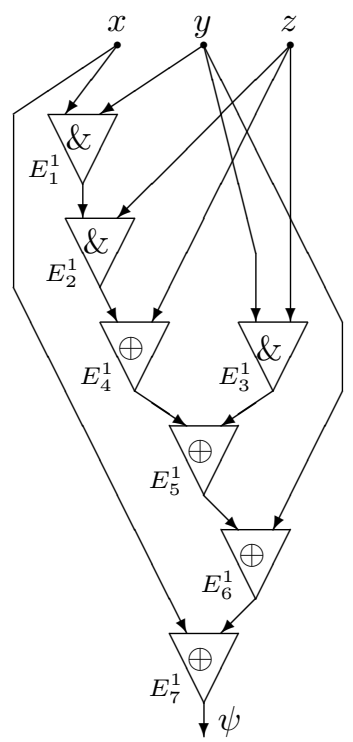

Рис. 1. Схема $\hat{S}_{B^{\prime}}^{\psi}$.

Лемма 8. Для схемы $\hat{S}_{B^{\prime}}^{\psi}$, изображенной на рисунке 1 , множество входных наборов $A_{1}=\{(0,0,0),(1,1,1),(0,0,1)\}$ является единичным проверяющим тестом (относительно произвольных константных неисправностей на выходах элементов).

Доказательство. Построим таблицу, в которой опишем поведения всех элементов схемы (обозначения элементов соответствуют рисунку 1) на наборах множества $A_{1}$ при отсутствии неисправностей в схеме. Именно, строкам таблицы соответствуют различные наборы из множества $A_{1}$, а столбцам - элементы схемы; на пересечении строки и столбца указываются три значения - значения на двух входах (упорядоченных слева направо) и на выходе данного элемента на данном наборе:

\begin{tabular}{|c||c|c|c|c|c|c|c|}
\hline$(x, y, z)$ & $E_{1}^{1}$ & $E_{2}^{1}$ & $E_{3}^{1}$ & $E_{4}^{1}$ & $E_{5}^{1}$ & $E_{6}^{1}$ & $E_{7}^{1}$ \\
\hline \hline$(0,0,0)$ & 000 & 000 & 000 & 000 & 000 & 000 & 000 \\
$(1,1,1)$ & 111 & 111 & 111 & 110 & 011 & 110 & 101 \\
$(0,0,1)$ & 000 & 010 & 010 & 011 & 101 & 101 & 011 \\
\hline
\end{tabular}

Из таблицы по лемме 7 сразу следует, что константные неисправности на выходах элементов с $E_{2}^{1}$ по $E_{7}^{1}$ обнаруживаются на множестве наборов $A_{1}$ (заметим, что для обнаружения неисправностей на выходах элементов $E_{2}^{1}$ и $E_{3}^{1}$ достаточно взять два набора $(0,0,0)$ и $(1,1,1))$. Рассмотрим элемент $E_{1}^{1}$. Пусть на его выходе произошла неисправность типа „константа 0 “. Тогда на наборе $(1,1,1)$ на выходах элементов $E_{1}^{1}, E_{2}^{1}$ появятся „неправильные“ значения 0, и далее вдоль цепочки элементов с 
$E_{4}^{1}$ по $E_{7}^{1}$ все значения на выходах элементов будут „неправильными“, и указанная неисправность будет обнаружена. Пусть на выходе элемента $E_{1}^{1}$ произошла неисправность типа „константа 1 “. Тогда на наборе $(0,0,1)$ на выходах элементов $E_{1}^{1}, E_{2}^{1}$ появятся „неправильные“ значения 1, и (аналогично предыдущему случаю) указанная неисправность будет обнаружена. Лемма доказана.

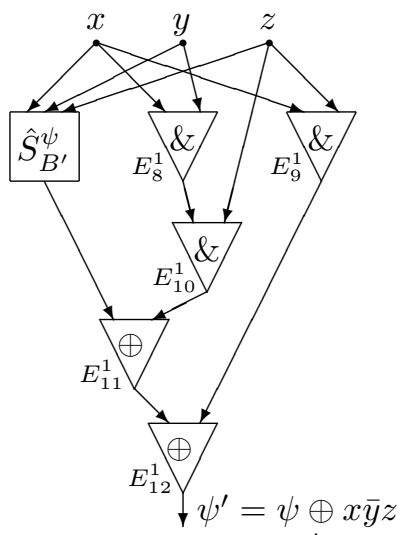

Рис. 2. Схема $\hat{S}_{B^{\prime}}^{\psi^{\prime}}$.

Лемма 9. Для схемъ $\hat{S}_{B^{\prime}}^{\psi^{\prime}}$, изображенной на рисунке 2 , множество входных наборов $A_{1}=\{(0,0,0),(1,1,1),(0,0,1)\}$ является единичным проверяюшим тестом (относительно произвольных константных неисправностей на выходах элементов).

Доказательство аналогично доказательству предыдущей леммы. Из рисунка 2 ясно, что для доказательства леммы 9 достаточно использовать таблицу из доказательства леммы 8 , достроив ее для элементов с $E_{8}^{1}$ по $E_{12}^{1}$ :

\begin{tabular}{|c||c|c|c|c|c|}
\hline$(x, y, z)$ & $E_{8}^{1}$ & $E_{9}^{1}$ & $E_{10}^{1}$ & $E_{11}^{1}$ & $E_{12}^{1}$ \\
\hline \hline$(0,0,0)$ & 000 & 000 & 000 & 000 & 000 \\
$(1,1,1)$ & 111 & 111 & 111 & 110 & 011 \\
$(0,0,1)$ & 000 & 010 & 010 & 101 & 101 \\
\hline
\end{tabular}

Из таблицы по лемме 7 сразу следует, что константные неисправности на выходах элементов с $E_{2}^{1}$ по $E_{7}^{1}$ и с $E_{9}^{1}$ по $E_{12}^{1}$ обнаруживаются на множестве наборов $A_{1}$ (заметим, что для обнаружения неисправностей на выходах элементов $E_{2}^{1}, E_{3}^{1}, E_{9}^{1}$ и $E_{10}^{1}$ достаточно взять два набора $(0,0,0)$ и $\left.(1,1,1)\right)$. Элементы $E_{1}^{1}$ и $E_{8}^{1}$ рассматриваются в полной аналогии с элементом $E_{1}^{1}$ в лемме 8 . Таким образом, все константные неисправности на выходах элементов в схеме $\hat{S}_{B^{\prime}}^{\psi^{\prime}}$ будут обнаружены. Лемма доказана.

Лемма 10. Для схемъ $\hat{S}_{B^{\prime \prime}}^{\psi}$, изображенной на рисунке 3 , множество входных наборов $A_{1}=\{(0,0,0),(1,1,1),(0,0,1)\}$ является единичным проверяющим тестом (относительно произволъных константных неисправностей на выходах элементов).

Доказательство. Построим для схемы $\hat{S}_{B^{\prime \prime}}^{\psi}$ таблицу, аналогичную таблице из доказательства леммы 8 (в столбцах, соответствующих элементам с одним входом, указывается значение на входе и на выходе элемента): 


\begin{tabular}{|c||c|c|c|c|c|c|c|c|c|}
\hline$(x, y, z)$ & $E_{1}^{2}$ & $E_{2}^{2}$ & $E_{3}^{2}$ & $E_{4}^{2}$ & $E_{5}^{2}$ & $E_{6}^{2}$ & $E_{7}^{2}$ & $E_{8}^{2}$ & $E_{9}^{2}$ \\
\hline \hline$(0,0,0)$ & 01 & 010 & 010 & 01 & 010 & 000 & 000 & 010 & 000 \\
$(1,1,1)$ & 10 & 101 & 101 & 10 & 101 & 110 & 011 & 100 & 101 \\
$(0,0,1)$ & 01 & 010 & 110 & 01 & 110 & 011 & 101 & 111 & 011 \\
\hline
\end{tabular}

Из таблицы по лемме 7 сразу следует, что константные неисправности на выходах элементов с $E_{5}^{2}$ по $E_{9}^{2}$ и $E_{3}^{2}$ обнаруживаются на множестве наборов $A_{1}$. Ясно, что для обнаружения константной неисправности $\sigma$ на выходе элемента $E_{2}^{2}$ достаточно обнаружить константную неисправность $\bar{\sigma}$ на выходе элемента $E_{4}^{2}$. Пусть на выходе $E_{4}^{2}$ произошла неисправность типа „константа 0“. Тогда на наборе $(0,0,1)$ на выходах элементов с $E_{5}^{2}$ по $E_{9}^{2}$ появятся „неправильные“ значения, и (аналогично случаю с $E_{1}^{1}$ из леммы 8) указанная неисправность будет обнаружена. Пусть на выходе $E_{4}^{2}$ произошла неисправность типа „константа $1 “$. Тогда на наборе $(1,1,1)$ на выходах элементов с $E_{5}^{2}$ по $E_{9}^{2}$ появятся „неправильные“ значения, и указанная неисправность также будет обнаружена.

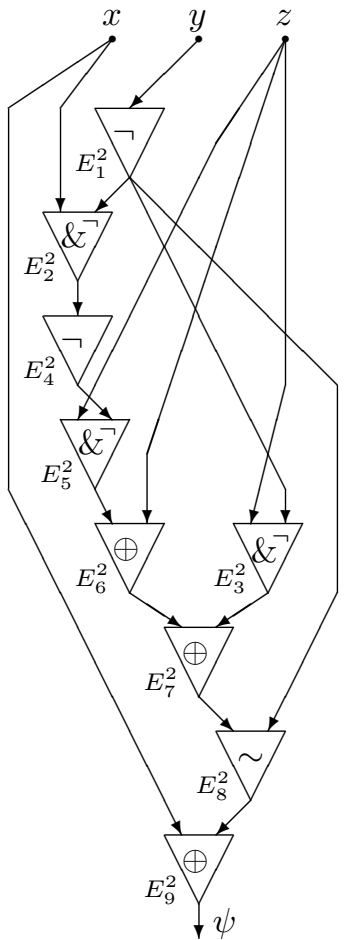

Рис. 3. Схема $\hat{S}_{B^{\prime \prime}}^{\psi}$.

Рассмотрим элемент $E_{1}^{2}$. Построим (аналогичную предыдущей таблице) таблицу значений на входах и выходах элементов схемы $\hat{S}_{B^{\prime \prime}}^{\psi}$ на наборе $(0,0,0)$ при наличии неисправности типа „константа $0 “$ на выходе элемента $E_{1}^{2}$ :

\begin{tabular}{|c||c|c|c|c|c|c|c|c|c|}
\hline$(x, y, z)$ & $E_{1}^{2}$ & $E_{2}^{2}$ & $E_{3}^{2}$ & $E_{4}^{2}$ & $E_{5}^{2}$ & $E_{6}^{2}$ & $E_{7}^{2}$ & $E_{8}^{2}$ & $E_{9}^{2}$ \\
\hline \hline$(0,0,0)$ & 00 & 000 & 000 & 01 & 010 & 000 & 000 & 001 & 011 \\
\hline
\end{tabular}

Последняя цифра в этой таблице (значение на выходе схемы) при сравнении с последней цифрой в строке для набора $(0,0,0)$ в предыдущей таблице показывает, что на данном наборе указанная неисправность обнаруживается. Построим теперь таблицу значений на входах и выходах элементов исследуемой схемы на наборе $(1,1,1)$ при наличии неисправности типа „константа 1 “ на выходе элемента $E_{1}^{2}$ : 


\begin{tabular}{|c||c|c|c|c|c|c|c|c|c|}
\hline$(x, y, z)$ & $E_{1}^{2}$ & $E_{2}^{2}$ & $E_{3}^{2}$ & $E_{4}^{2}$ & $E_{5}^{2}$ & $E_{6}^{2}$ & $E_{7}^{2}$ & $E_{8}^{2}$ & $E_{9}^{2}$ \\
\hline \hline$(1,1,1)$ & 11 & 110 & 110 & 01 & 110 & 011 & 101 & 111 & 110 \\
\hline
\end{tabular}

Последняя цифра в таблице при сравнении с последней цифрой в строке для набора $(0,0,0)$ в первой таблице из доказательства этой леммы показывает, что на данном наборе указанная неисправность обнаруживается. Лемма доказана.

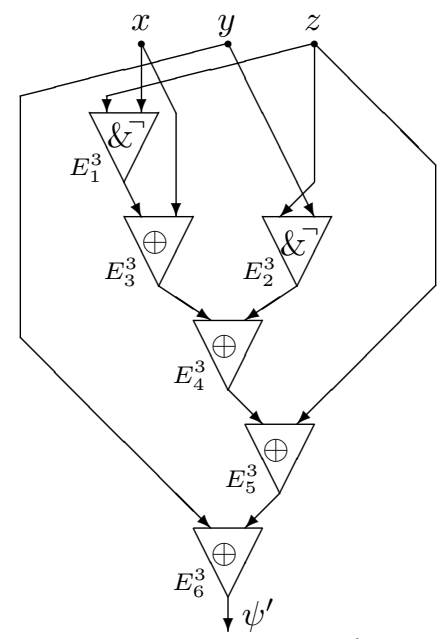

Рис. 4. Схема $\hat{S}_{B^{\prime \prime}}^{\psi^{\prime}}$

Лемма 11. Для схемъ $\hat{S}_{B^{\prime \prime}}^{\psi^{\prime}}$, изображенной на рисунке 4 , множество входных наборов $A_{1}=\{(0,0,0),(1,1,1),(0,0,1)\}$ является единичным проверяющим тестом (относительно произвольных константных неисправностей на выходах элементов).

Доказательство аналогично доказательству лемм 8-10. Построим для схемы $\hat{S}_{B^{\prime \prime}}^{\psi^{\prime}}$ при отсутствии неисправностей таблицу, аналогичную таблице из доказательства леммы 8:

\begin{tabular}{|c||c|c|c|c|c|c|}
\hline$(x, y, z)$ & $E_{1}^{3}$ & $E_{2}^{3}$ & $E_{3}^{3}$ & $E_{4}^{3}$ & $E_{5}^{3}$ & $E_{6}^{3}$ \\
\hline \hline$(0,0,0)$ & 000 & 000 & 000 & 000 & 000 & 000 \\
$(1,1,1)$ & 110 & 110 & 011 & 101 & 110 & 101 \\
$(0,0,1)$ & 101 & 101 & 101 & 110 & 011 & 011 \\
\hline
\end{tabular}

Из таблицы по лемме 7 сразу следует, что константные неисправности на выходах всех элементов схемы обнаруживаются на множестве наборов $A_{1}$. Лемма доказана.

Лемма 12. Для схемы $\hat{S}_{B_{1}}^{\sim}$, изображенной на рисунке 5 , множество входных наборов $A_{2}=\{(0,0),(0,1),(1,0)\}$ является единичным проверяющим тестом (относительно произвольных константных неисправностей на выходах элементов). 


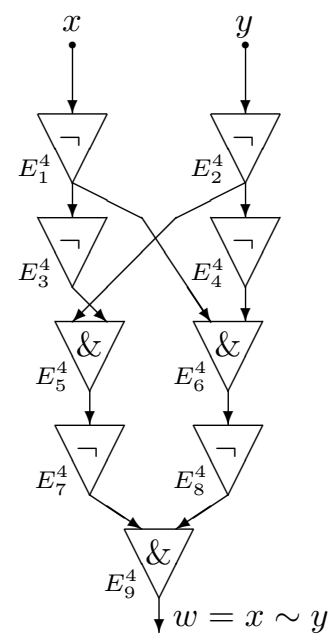

Рис. 5. Схема $\hat{S}_{B_{1}}$.

Доказательство аналогично доказательству лемм 8-11. Построим для схемы $\hat{S}_{B_{1}}^{\sim}$ при отсутствии неисправностей таблицу, аналогичную первой таблице из доказательства леммы 10:

\begin{tabular}{|c||c|c|c|c|c|c|c|c|c|}
\hline$(x, y)$ & $E_{1}^{4}$ & $E_{2}^{4}$ & $E_{3}^{4}$ & $E_{4}^{4}$ & $E_{5}^{4}$ & $E_{6}^{4}$ & $E_{7}^{4}$ & $E_{8}^{4}$ & $E_{9}^{4}$ \\
\hline \hline$(0,0)$ & 01 & 01 & 10 & 10 & 100 & 100 & 01 & 01 & 111 \\
$(0,1)$ & 01 & 10 & 10 & 01 & 000 & 111 & 01 & 10 & 100 \\
$(1,0)$ & 10 & 01 & 01 & 10 & 111 & 000 & 10 & 01 & 010 \\
\hline
\end{tabular}

Заметим, что при преобразовании симметрии изображенной на рисунке 5 схемы относительно вертикальной прямой, проходящей посередине выходного элемента схемы и последующей транспозиции входов $x$ и $y$ схема не изменится, а множество $A_{2}$ перейдет в себя. Поэтому достаточно доказать, что на множестве $A_{2}$ обнаруживаются все константные неисправности элементов с нечетными нижними индексами. Для элемента $E_{9}^{4}$ это напрямую следует из анализа последнего столбца в таблице. Ясно, что для обнаружения константной неисправности $\sigma$ на выходе элемента $E_{5}^{4}$ достаточно обнаружить константную неисправность $\bar{\sigma}$ на выходе элемента $E_{7}^{4}$. Но неисправность типа „константа 0“ на выходе элемента $E_{7}^{4}$ обнаруживается (как это следует из таблицы) на наборе $(0,0)$, а неисправность типа „константа 1 “ на выходе $E_{7}^{4}$ обнаруживается на наборе $(1,0)$. Неисправность типа „константа 0“ на выходе $E_{3}^{4}$ обнаруживается, так как на входном наборе $(1,0)$ на выходах элементов $E_{5}^{4}, E_{7}^{4}$, $E_{9}^{4}$ появятся значения 0, 1, 1 (соответственно). Неисправность типа „константа 1“ на выходе $E_{3}^{4}$ обнаруживается, так как на входном наборе $(0,0)$ на выходах элементов $E_{5}^{4}, E_{7}^{4}, E_{9}^{4}$ появятся значения $1,0,0$ (соответственно). Построим таблицу значений на входах и выходах элементов исследуемой схемы на наборе $(0,0)$ при наличии неисправности типа „константа 0“ на выходе элемента $E_{1}^{4}$ :

\begin{tabular}{|c||c|c|c|c|c|c|c|c|c|}
\hline$(x, y)$ & $E_{1}^{4}$ & $E_{2}^{4}$ & $E_{3}^{4}$ & $E_{4}^{4}$ & $E_{5}^{4}$ & $E_{6}^{4}$ & $E_{7}^{4}$ & $E_{8}^{4}$ & $E_{9}^{4}$ \\
\hline \hline$(0,0)$ & 00 & 01 & 01 & 10 & 111 & 000 & 10 & 01 & 010 \\
\hline
\end{tabular}

Видно, что указанная неисправность обнаруживается на наборе $(0,0)$. Построим теперь таблицу значений на входах и выходах элементов исследуемой схемы на наборе $(1,0)$ при наличии неисправности типа „константа $1 “$ на выходе элемента $E_{1}^{4}$ : 


\begin{tabular}{|c||c|c|c|c|c|c|c|c|c|}
\hline$(x, y)$ & $E_{1}^{4}$ & $E_{2}^{4}$ & $E_{3}^{4}$ & $E_{4}^{4}$ & $E_{5}^{4}$ & $E_{6}^{4}$ & $E_{7}^{4}$ & $E_{8}^{4}$ & $E_{9}^{4}$ \\
\hline \hline$(1,0)$ & 11 & 01 & 10 & 10 & 010 & 100 & 01 & 01 & 111 \\
\hline
\end{tabular}

Видно, что эта неисправность обнаруживается на наборе $(1,0)$. Лемма доказана.

Лемма 13. Для любой из бункиий $x \sim y, x \oplus y$ и для любой тройки А различных наборов из множества $A_{3}=\{(0,0),(0,1),(1,0),(1,1)\}$ найдется реализующая выбранную функцию неизбыточная схема $\hat{S}$ в базисе $B_{1}=\{\bar{x}, x \& y\}$, для которой множество наборов А является единичным проверяющим тестом (относительно произвольных константных неисправностей на выходах элементов).

Доказательство выводится из лемм 12, 4 и 5, а также того факта, что произвольная тройка наборов $A$ из условия настоящей леммы либо совпадает с тройкой наборов $A_{2}$ из леммы 12 , либо может быть получена из $A_{2}$ инвертированием одной или двух координат. Искомая схема $\hat{S}$ либо совпадает с $\hat{S}_{B_{1}}$, либо получается из нее „удалением“ одного или двух инцидентных входам инверторов (см. лемму 4), либо получается из таких схем добавлением к выходу схемы нового инвертора (с переносом выхода схемы на выход нового инвертора, см. лемму 5).

Лемма 14. Пусть множество $T$ входных наборов неизбыточной схемъ $S$ образует единичный проверяющий тест относительно произвольных константных неисправностей на выходах элементов в схеме $S$, и пусть $\Sigma_{E}-$ подсхема схемы $S$, имеющая один выход и эквивалентная (при отсутствии неисправностей) функииональному элементу $E$. Тогда схема $S^{\prime}$, полученная из $S$ эквивалентной подстановкой вместо подсхемы $\Sigma_{E}$ элемента $E$, неизбыточна, и множество наборов Т образует единичный проверяющий тест относительно произвольных константных неисправностей на выходах элементов в схеме $S^{\prime}$.

Доказательство этой леммы очевидно.

Лемма 15. Для любой из функций $x \sim y, x \oplus y$ и для любой тройки $А$ различных наборов из множества $A_{3}=\{(0,0),(0,1),(1,0),(1,1)\}$ найдется реализующая выбранную функцию неизбыточная схема $\hat{S}$ в базисе $B_{5}=\{\bar{x}, x \& \bar{y}\}$, для которой множество наборов А является единичным проверяющим тестом (относительно произвольных константных неисправностей на выходах элементов).

Доказательство легко выводится из лемм 12, 13 и 14.

\section{Синтез легкотестируемых неизбыточных схем в бази- $\operatorname{cax} B^{\prime}=\{x \& y, x \oplus y, x \sim y\}, B^{\prime \prime}=\{\bar{x}, x \& \bar{y}, x \oplus y, x \sim y\}$}

В данном разделе устанавливается, что функция Шеннона длины единичного проверяющего теста относительно произвольных константных неисправностей на выходах элементов в базисах $B^{\prime}=\{x \& y, x \oplus y, x \sim y\}$ и $B^{\prime \prime}=\{\bar{x}, x \& \bar{y}, x \oplus y, x \sim y\}$ не превосходит 4. Справедливо следующее утверждение.

Теорема 1. Для каждой булевой функиии $f\left(\tilde{x}^{n}\right)$ справедливы следующие утверждения:

1) существует реализующая функиию $f\left(\tilde{x}^{n}\right)$ неизбыточная $С Ф \vartheta S_{B^{\prime}}^{f}$ в базисе $B^{\prime}=\{x \& y, x \oplus y, x \sim y\}$, допускающая некоторый единичный проверяющий тест 
$\hat{T}_{n}$ длины, не превосходящей четырех, относительно произвольных константных неисправностей на выходах элементов;

2) и при этом, если число существенных переменных функиии $f$ не меньше двух, то для каждого функционального элемента Е суммы по модулю 2 или эквивалентности в схеме $S_{B^{\prime}}^{f}$ найдутся три набора из множества $\hat{T}_{n}$, на каждом из которых обнаруживается константная неисправность на выходе E, и при их подаче на входы схемы на входах элемента $E$ возникают три различных набора.

Доказательство. Пусть $f\left(\tilde{x}^{n}\right)$ - произвольная булева функция, зависящая (существенно или фиктивно) от переменных $x_{1}, x_{2}, \ldots, x_{n}$.

Перепишем полином Жегалкина функции $f$ в виде $P_{f}=K_{1} \oplus K_{2} \oplus \cdots \oplus K_{t} \oplus a_{0}$, где $K_{i}$ - конъюнкции различных переменных или переменные $(i=\overline{1, t}), a_{0} \in\{0,1\}$.

Случай 1. Пусть функция $f-$ нелинейная. Пусть, далее, $K_{1}-$ слагаемое минимального ранга в полиноме Жегалкина функции $f$, отличное от константы и переменной. Будем, не ограничивая общности, считать, что $K_{1}=x_{1} x_{2} \cdots x_{s}(2 \leqslant$ $s \leqslant n)$, и что слагаемые $K_{1}, K_{2}, \ldots, K_{q}(1 \leqslant q \leqslant t)$ - нелинейные, а слагаемые $K_{q+1}, K_{q+2}, \ldots, K_{t}$ - линейные (точнее, переменные).

Подслучай 1.1. Пусть функция $f$ существенно зависит ровно от двух переменных.
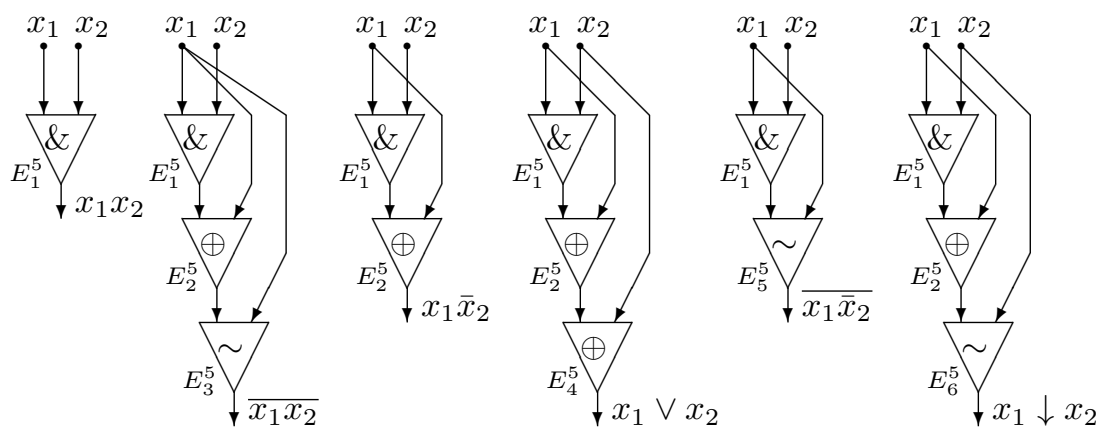

Рис. 6. Схемы $S_{B^{\prime}}^{f}$ для нелинейных функций двух переменных.

Положим $\hat{T}_{2}=\{(0,0),(1,0),(1,1)\}$.

Для функции $f=x_{1} x_{2}$ искомая схема состоит из одного конъюнктора. Для функции $f=x_{1} x_{2} \oplus 1$ искомая схема $S_{B^{\prime}}^{f}$ моделирует формулу $\left(x_{1} x_{2} \oplus x_{1}\right) \sim x_{1}$. Для функции $f \in\left\{x_{1} x_{2} \oplus x_{1},\left(x_{1} x_{2} \oplus x_{1}\right) \oplus x_{2}, x_{1} x_{2} \sim x_{1},\left(x_{1} x_{2} \oplus x_{1}\right) \sim x_{2}\right\}$ искомая схема $S_{B^{\prime}}^{f}$ моделирует ту формулу, которой эта функция здесь задается. Указанные схемы изображены на рисунке 6. (Остальные нелинейные функции двух переменных получаются из перечисленных функций транспозицией переменных, поэтому мы не будем их рассматривать.) Построим таблицы значений на входах и выходах элементов этих схем на наборах множества $\hat{T}_{2}$ (как в лемме 8):

\begin{tabular}{|c||c|c|c|c|c|c|}
\hline$(x, y)$ & $E_{1}^{5}$ & $E_{2}^{5}$ & $E_{3}^{5}$ & $E_{4}^{5}$ & $E_{5}^{5}$ & $E_{6}^{5}$ \\
\hline \hline$(0,0)$ & 000 & 000 & 001 & 000 & 001 & 001 \\
$(1,0)$ & 100 & 011 & 111 & 101 & 010 & 100 \\
$(1,1)$ & 111 & 110 & 010 & 011 & 111 & 010 \\
\hline
\end{tabular}

Из таблицы по лемме 7 сразу следует, что константные неисправности на выходах всех элементов этих схем обнаруживаются на множестве наборов $\hat{T}_{2}$ (то есть множество наборов $\hat{T}_{2}$ образует единичный проверяющий тест для каждой из построенных 
схем). Вторая часть утверждения теоремы в настоящем подслучае проверяется по построенной таблице непосредственно.

Подслучай 1.2. Пусть функция $f$ существенно зависит не менее чем от трех переменных.

Подслучай 1.2.1. Пусть функция $f$ такова, что в $P_{f}$ нет линейных слагаемых, входящих в качестве множителей-переменных в $K_{1}$. Построим неизбыточную схему $S_{B^{\prime}}^{f}$, реализующую $f$ и допускающую единичный проверяющий тест длины 4 относительно константных неисправностей на выходах элементов.

Схема $S_{B^{\prime}}^{f}$ составлена из блоков $S_{1}, S_{2}, S_{3}, S_{4}$, как показано на рисунке 7.

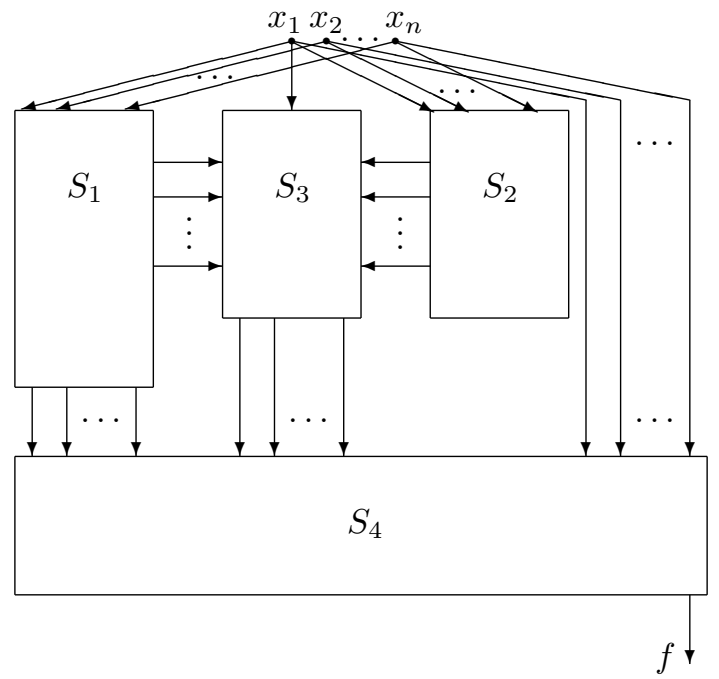

Рис. 7. Блочное устройство схем $S_{B^{\prime}}^{f}, S_{B^{\prime \prime}}^{f}$ для нелинейной функции, существенно зависящей от не менее чем трех переменных.

Блок $S_{1}$ представляет собой $q$ цепочек конъюнкторов $C_{1}, C_{2}, \ldots, C_{q}$, моделирующих все нелинейные слагаемые $K_{1}, K_{2}, \ldots, K_{q}$ (соответственно) полинома Жегалкина $P_{f}$ функции $f$. При этом на входы первого элемента каждой цепочки подаются переменные (в частности, на входы первого элемента цепочки $C_{1}$ подаются переменные $\left.x_{1}, x_{2}\right)$; на левый вход каждого конъюнктора цепочки, начиная со второго, подается выход предыдущего конъюнктора; на правые входы конъюнкторов цепочки, начиная со второго, подаются входные переменные. Так как $K_{1}$ - самая короткая конъюнкция (отличная от переменной), то для любого $i \in\{2,3, \ldots, q\}$ в конъюнкции $K_{i}$ имеется переменная $x_{j_{i}}$, не входящая в $K_{1}$. Потребуем, чтобы на правый вход первого конъюнктора цепочки $C_{i}$ подавалась бы переменная $x_{j_{i}}$, а на левый вход первого конъюнктора цепочки $C_{i}$ - переменная $x_{1}$, если она входит в $K_{i}$, и любая переменная конъюнкции $K_{i}$, отличная от $x_{j_{i}}$, если $x_{1}$ не входит в $K_{i}$.

Блок $S_{2}$ представляет собой копию блока $S_{1}$, в которой удалены последние элементы в каждой из цепочек $C_{1}, C_{2}, \ldots, C_{q}$. Пусть число элементов в $S_{2}$ есть $L_{2}$, обозначим элементы из $S_{2}$ через $E_{1}^{0}, E_{2}^{0}, \ldots, E_{L_{2}}^{0}$, считая, что в начале этого перечня следуют конъюнкторы из копии цепочки $C_{1}$ в соответствии с их порядком в цепочке, затем идут конъюнкторы из копии цепочки $C_{2}$ в соответствии с их порядком в цепочке, и так далее, - завершают список конъюнкторы из копии цепочки $C_{q}$ в соответствии с их порядком в цепочке. Если полином Жегалкина функции $f$ имеет 
степень (максимальный ранг слагаемого) два, то $L_{2}=0$, и блок $S_{2}$ не содержит элементов.

Блок $S_{3}$ состоит из трехвходовых подсхем $\Sigma_{i}^{3}\left(i=\overline{1, L_{2}}\right)$, состоящих из функциональных элементов базиса $B^{\prime}$ (при $L_{2}=0$ блок $S_{3}$ не содержит элементов). Для каждой пары „дублирующих друг друга“ конъюнкторов из блоков $S_{1}$ и $S_{2}$ строится одна такая подсхема. Для каждого конъюнктора $E_{i}^{2}=E_{\&}^{\prime \prime}$ из блока $S_{2}$, являющегося выходным элементом цепочки из блока $S_{2}$, и его копии $E_{\&}^{\prime}$ в блоке $S_{1}$ соответствующая подсхема $\Sigma_{i}^{3}$ представляет собой изображенную на рисунке 1 трехвходовую схему $\hat{S}_{B^{\prime}}^{\psi}$, реализующую функцию $\psi(x, y, z)$; на левый вход схемы $\hat{S}_{B^{\prime}}^{\psi}$ подается выход элемента $E_{\&}^{\prime}$, на средний вход - выход элемента $E_{\&}^{\prime \prime}$, а на правый - переменная $x_{1}$. Для каждого конъюнктора $E_{i}^{2}=E_{\&}^{\prime \prime}$ из блока $S_{2}$, отличного от выходного элемента цепочки из блока $S_{2}$, и его копии $E_{\&}^{\prime}$ в блоке $S_{1}$ соответствующая подсхема $\Sigma_{i}^{3}$ представляет собой трехвходовую схему $\hat{S}_{B^{\prime}}^{\psi^{\prime}}$, изображенную на рисунке 2 (подсхема $\hat{S}_{B^{\prime}}^{\psi}$ определена выше); на левый вход этой подсхемы подается выход элемента $E_{\&}^{\prime}$, на средний вход $\Sigma_{i}^{3}$ - выход элемента $E_{\&}^{\prime \prime}$, а на правый - переменная $x_{1}$. Заметим, что на выходе каждой подсхемы $\Sigma_{i}^{3}$ при отсутствии неисправностей реализуется функция $x_{1}$.

Блок $S_{4}$ состоит из цепочки $C$ элементов сумм по модулю 2 и, быть может, одного функционального элемента типа „эквивалентность“. При этом на левый вход самого первого элемента цепочки $C$ подается выход цепочки $C_{1}$ блока $S_{1}$; на левый вход каждого элемента цепочки $C$, начиная со второго, подается выход предыдущего элемента этой цепочки; на правые входы первых $(q-1)$ элементов цепочки $C$ подаются выходы цепочек $C_{2}, \ldots, C_{q}$ блока $S_{1}$ (в указанном порядке); на правые входы следующих $L_{2}$ элементов цепочки $C$ подаются выходы всех трехвходовых подсхем $\Sigma_{1}^{3}$, $\Sigma_{2}^{3}, \ldots, \Sigma_{L_{2}}^{3}$ из блока $S_{3}$ в указанном порядке (и, если $L_{2}$ нечетное, то на правый вход следующего элемента цепочки $C$ подается переменная $x_{1}$ ), на правые входы следующих $t-q$ элементов цепочки $C$ подаются переменные, являющиеся линейными слагаемыми в $P_{f}$, в порядке, соответствующем возрастанию индексов этих переменных. Если $a_{0}=0$, то все элементы цепочки $C$ являются элементами сумм по модулю 2 , и других элементов в блоке $S_{4}$ нет. Если же $a_{0}=1$, то в $C$ последним элементом (обозначим его через $E_{\sim}$ ) является элемент типа „эквивалентность“, а остальными элементами цепочки $C$ являются элементы сумм по модулю 2. Обозначим через $D_{1}$ последовательность всех элементов цепочки $C$ (в порядке следования в цепочке), у которых хотя бы на один вход подается выход цепочки из блока $S_{1}$. Пусть, далее, $D_{2}-$ последовательность всех элементов цепочки $C$ (в порядке следования в цепочке), у которых на правый вход подается выход элемента из блока $S_{3}$ (если $L_{2}$ нечетное, то к $D_{2}$ относится и следующий функциональный элемент суммы по модулю 2 , на вход которого подается переменная $x_{1}$; таким образом, в $D_{2}$ четное число элементов). Наконец, пусть $D_{3}-$ последовательность всех элементов цепочки $C$ (в порядке следования в цепочке), у которых на правый вход подается входная переменная. Заметим, что при $q=1$ у $D_{1}$ и $D_{2}$ есть один общий элемент, а в остальных случаях попарно общих элементов у $D_{1}, D_{2}$ и $D_{3}$ нет; последовательности $D_{2}$ и $D_{3}$ могут не содержать элементов. Отметим, что цепочка $C$ содержит хотя бы один элемент.

Выход последнего элемента цепочки $C$ является выходом схемы $S_{B^{\prime}}^{f}$.

Легко видеть, что при отсутствии неисправностей схема $S_{B^{\prime}}^{f}$ реализует функцию $f$ (так как, в силу построения, в схеме $S_{B^{\prime}}^{f}$ по модулю 2 суммируются слагаемые поли- 
нома Жегалкина функции $f$ по одному разу каждое, а также четное число равных функций $\left.x_{1}\right)$.

Положим $\hat{T}_{n}=\left\{\left(\tilde{0}^{n}\right),\left(\tilde{1}^{n}\right),\left(1, \tilde{0}^{n-1}\right),\left(\tilde{1}^{s}, \tilde{0}^{n-s}\right)\right\}$. Докажем, что $\hat{T}_{n}-$ единичный проверяющий тест для схемы $S_{B^{\prime}}^{f}$.

На наборе $\left(\tilde{0}^{n}\right)$ на выходах всех конъюнкторов из блоков $S_{1}, S_{2}$ будут нули, на входах каждой подсхемы $\Sigma_{i}^{3}\left(i=\overline{1, L_{2}}\right)$ возникнут наборы $(0,0,0)$. На наборе $\left(\tilde{1}^{n}\right)$ на выходах всех конъюнкторов из блоков $S_{1}, S_{2}$ будут единицы, на входах каждой подсхемы $\Sigma_{i}^{3}$ возникнут наборы $(1,1,1)$. На наборе $\left(1, \tilde{0}^{n-1}\right)$ на выходах всех конъюнкторов из блоков $S_{1}, S_{2}$ будут нули, на входах каждой подсхемы $\Sigma_{i}^{3}$ возникнут наборы $(0,0,1)$. Множество $\{(0,0,0),(1,1,1),(0,0,1)\}$ образует единичный проверяющий тест для каждой из схем $\hat{S}_{B^{\prime}}^{\psi}, \hat{S}_{B^{\prime}}^{\psi^{\prime}}$ по леммам 8 и 9 . В силу устройства схем $S_{B^{\prime}}^{f}, \hat{S}_{B^{\prime}}^{\psi}, \hat{S}_{B^{\prime}}^{\psi^{\prime}}$ каждый элемент суммы по модулю 2 и эквивалентности в схеме $S_{B^{\prime}}^{f}$ имеет неветвящийся выход и лежит на продолжающейся до выхода схемы $S_{B^{\prime}}^{f}$ цепи, состоящей только из элементов суммы по модулю 2 и эквивалентности. Значит, в силу леммы 7 любая константная неисправность выходного элемента (а это элемент суммы по модулю 2) любой подсхемы $\Sigma_{i}^{3}$ будет обнаружена на множестве входных наборов $\hat{T}_{n}$ схемы $S_{B^{\prime}}^{f}$. Поэтому по леммам $2,7,8$ и 9 на наборах из $\hat{T}_{n}$ будут обнаруживаться все константные неисправности всех элементов, лежащих в подсхемах $\Sigma_{i}^{3}$ схемы $S_{B^{\prime}}^{f}$. Заметим, что для всех элементов суммы по модулю 2 и эквивалентности, лежащих в подсхемах $\Sigma_{i}^{3}$ схемы $S_{B^{\prime}}^{f}$, второе утверждение настоящей теоремы выполняется, как это непосредственно следует из анализа таблиц из доказательств лемм 8-9 и из леммы 7.

Легко видеть, что на наборе $\left(\tilde{0}^{n}\right)$ обнаруживаются все неисправности типа „константа 1“ на выходах всех остальных элементов схемы $S_{B^{\prime}}^{f}$, кроме $E_{\sim}$, а также обнаруживается неисправность типа „константа 0“ на выходе $E_{\sim}$ (если этот элемент есть в $\left.S_{B^{\prime}}^{f}\right)$. Действительно, $f\left(\tilde{0}^{n}\right)=a_{0}$, и на выходах всех элементов схемы $S_{B^{\prime}}^{f}$ (при отсутствии в ней неисправностей), кроме $E_{\sim}$, на наборе $\left(\tilde{0}^{n}\right)$ оказываются нули. Если неисправность типа „1“ произошла на выходе конъюнктора $E_{\&}^{\prime}$ блока $S_{1}$, не являющегося последним конъюнктором одной из цепочек $C_{1}, C_{2}, \ldots, C_{q}$, то на выходе следующего за $E_{\&}^{\prime}$ конъюнктора в цепочке будет по-прежнему 0 , и на выходе цепочки конъюнкторов, в которую входит $E_{\&}^{\prime}$, будет по-прежнему 0. Зато на выходе трехвходовой подсхемы $\Sigma_{i}^{3}$, на вход которой подается выход $E_{\&}^{\prime}$, появится 1 , и на выходе схемы $S_{B^{\prime}}^{f}$ значение изменится с $a_{0}$ на $\bar{a}_{0}$. Если неисправность типа „1“ произошла на выходе конъюнктора $E_{\&}^{\prime \prime}$ блока $S_{2}$, то на выходе следующих за $E_{\&}^{\prime \prime}$ конъюнкторов (если они есть) в цепочке будут по-прежнему 0. Зато на выходе трехвходовой подсхемы $\Sigma_{i}^{3}$, на вход которой подается выход $E_{\&}^{\prime \prime}$, появится 1 , и на выходе схемы $S_{B^{\prime}}^{f}$ значение изменится с $a_{0}$ на $\bar{a}_{0}$. Если же неисправность типа „1“ произошла на выходе какого-то другого элемента схемы $S_{B^{\prime}}^{f}$ (кроме $E_{\sim}$, элементов из $S_{3}$ и упомянутых выше конъюнкторов), то, в силу того, что выход этого элемента не ветвится и подается на вход идущей до выхода схемы цепочки элементов сумм по модулю 2 и эквивалентности, значение на выходе схемы $S_{B^{\prime}}^{f}$ изменится с $a_{0}$ на $\bar{a}_{0}$. Так же дело обстоит с неисправностью типа „0“ на выходе $E_{\text { }}$.

На наборе $\left(\tilde{1}^{n}\right)$ обнаруживаются все неисправности типа „0“ на выходах всех конъюнкторов схемы $S_{B^{\prime}}^{f}$, не лежащих в $S_{3}$. Действительно, на наборе $\left(\tilde{1}^{n}\right)$ на выходах всех конъюнкторов и трехвходовых подсхем $\Sigma_{i}^{3}\left(i \in\left\{1, \ldots, L_{2}\right\}\right)$ схемы $S_{B^{\prime}}^{f}$ (при отсутствии в ней неисправностей) оказываются единицы. Неисправность типа „0“ на выходе конъюнктора $E_{\&}^{\prime}$ блока $S_{1}$, не являющегося последним конъюнктором це- 
почки $C_{i}(i \in\{1,2, \ldots, q\})$, будет обнаружена на наборе $\left(\tilde{1}^{n}\right)$, поскольку значение на выходе цепочки конъюнкторов, в которую входит $E_{\&}^{\prime}$ (и только этой цепочки конъюнкторов), изменится с 1 на 0, а значения на выходах всех трехвходовых подсхем $\Sigma_{i}^{3}$ останутся равными 1 . Неисправность типа „0“ на выходе конъюнктора $E_{\&}^{\prime \prime}$ блока $S_{2}$ будет обнаружена на наборе $\left(\tilde{1}^{n}\right)$, поскольку значение на выходе трехвходовой подсхемы $\Sigma_{i}^{3}$, на средний вход которой подается выход последнего элемента из той цепочки конъюнкторов блока $S_{2}$, которой принадлежит $E_{\&}^{\prime \prime}$, изменится с 1 на 0 , а значения на выходах остальных трехвходовых подсхем $\Sigma_{j}^{3}$ останутся равными 1 . Если же неисправность типа „0“ произошла на выходе последнего конъюнктора цепочки $C_{i}$, то, в силу того, что выход этого элемента не ветвится и подается на вход идущей до выхода схемы цепочки элементов сумм по модулю 2 и эквивалентности, значение на выходе схемы $S_{B^{\prime}}^{f}$ на этом наборе изменится.

На наборе $\left(1, \tilde{0}^{n-1}\right)$ на выходах всех конъюнкторов схемы $S_{B^{\prime}}^{f}$ (при отсутствии неисправностей) оказываются нули, на выходах всех трехвходовых подсхем $\Sigma_{i}^{3}-$ единицы.

На наборе $\left(\tilde{1}^{s}, \tilde{0}^{n-s}\right)$ (при отсутствии в схеме неисправностей) на выходах всех конъюнкторов цепочки $C_{1}$ блока $S_{1}$ и на выходах копий этих конъюнкторов в блоке $S_{2}$ оказываются единицы, на выходах всех остальных конъюнкторов схемы $S_{B^{\prime}}^{f}$ вне блока $S_{3}$ - нули (поскольку на правые входы первых конъюнкторов других цепочек конъюнкторов подаются переменные, не входящие в $K_{1}$ и принимающие на данном наборе значение 0), на выходах всех трехвходовых подсхем $\Sigma_{i}^{3}$ оказываются единицы (поскольку на правые входы трехвходовых подсхем $\Sigma_{i}^{3}$ подается переменная $x_{1}$, принимающая на данном наборе значение 1).

Изучим более подробно, какие неисправности элементов цепочки $C$ обнаруживаются на наборах множества $\hat{T}_{n}$, составив таблицы, аналогичные таблице из доказательства леммы 8 (таблицы значений на входах и выходах элементов цепочки $C$ при отсутствии неисправностей в схеме). Отметим, что по лемме 7 при отсутствии неисправностей в схеме наличие значения $\gamma$ на выходе элемента $E$ цепочки $C$ на входном наборе $\tilde{\alpha}$ из $\hat{T}_{n}$ влечет обнаружение на наборе $\tilde{\alpha}$ неисправности типа „, $\bar{\gamma}^{*}$ на выходе элемента $E$. Условимся при построении таблиц, что выходным элементом схемы $S_{B^{\prime}}^{f}$ далее в этом и следующем подслучаях (1.2.1 и 1.2 .2 и 2.4) является элемент суммы по модулю 2 (это не сильно ограничивает общность, так как если бы выходным элементом был бы единственнный элемент эквивалентности в цепочке $C$, то это привело бы лишь к инвертированию всех третьих координат в столбце для находящегося на его месте выходного элемента суммы по модулю 2). Для построения таблиц будут использоваться (по умолчанию) изложенные выше сведения о значениях последних элементов цепочек блока $S_{1}$ и выходных элементов подсхем $\Sigma_{i}^{3}\left(i \in\left\{1, \ldots, L_{2}\right\}\right)$ схемы $S_{B^{\prime}}^{f}$ при отсутствии в ней неисправностей на наборах из $\hat{T}_{n}$.

При построении таблицы удобно рассмотреть два разных подслучая (следует помнить, что в цепочке $D_{2}$ всегда четное число элементов).

Подслучай 1.2.1.1. Число $q$ - нечетное. Столбец $Q_{1}$ соответствует элементам цепочки $D_{1}$ с нечетными (порядковыми) номерами при $q>1$ (при $q=1$ единственный такой элемент является первым элементом цепочки $\left.D_{2}\right)$, столбец $Q_{2}$ соответствует элементам цепочки $D_{1}$ с четными номерами, столбец $Q_{3}$ соответствует элементам цепочки $D_{2}$ с нечетными номерами, столбец $Q_{4}$ соответствует элементам цепочки $D_{2}$ с четными номерами, столбец $Q_{5}$ соответствует элементам цепочки $D_{3}$ 
с нечетными номерами, столбец $Q_{6}$ соответствует элементам цепочки $D_{3}$ с четными номерами:

\begin{tabular}{|ccccccc||c|c|c|c|c|c|}
\hline$\left(x_{1}\right.$, & $x_{2}$, & $\ldots$, & $x_{s}$, & $x_{s+1}$, & $\ldots$, & $\left.x_{n}\right)$ & $Q_{1}$ & $Q_{2}$ & $Q_{3}$ & $Q_{4}$ & $Q_{5}$ & $Q_{6}$ \\
\hline \hline$(0$, & 0, & $\ldots$, & 0, & 0, & $\ldots$, & $0)$ & 000 & 000 & 000 & 000 & 000 & 000 \\
$(1$, & 0, & $\ldots$, & 0, & 0, & $\ldots$, & $0)$ & 000 & 000 & 011 & 110 & 000 & 000 \\
$(1$, & 1, & $\ldots$, & 1, & 0, & $\ldots$, & $0)$ & 101 & 101 & 110 & 011 & 101 & 101 \\
$(1$, & 1, & $\ldots$, & 1, & 1, & $\ldots$, & $1)$ & 110 & 011 & 110 & 011 & 110 & 011 \\
\hline
\end{tabular}

Как видно из таблицы (с учетом леммы 7), все константные неисправности элементов цепочки $C$ обнаруживаются на множестве $\hat{T}_{n}$ и второе утверждение теоремы выполняется для всех этих элементов.

Подслучай 1.2.1.2. Число $q$ - четное. Столбец $Q_{1}$ соответствует элементам цепочки $D_{1}$ с нечетными номерами, столбец $Q_{2}$ соответствует элементам цепочки $D_{1}$ с четными номерами, столбец $Q_{7}$ соответствует элементам цепочки $D_{2}$ с нечетными номерами, столбец $Q_{8}$ соответствует элементам цепочки $D_{2}$ с четными номерами, столбец $Q_{6}$ соответствует элементам цепочки $D_{3}$ с нечетными номерами, столбец $Q_{5}$ соответствует элементам цепочки $D_{3}$ с четными номерами:

\begin{tabular}{|ccccccc||c|c|c|c|c|c|}
\hline$\left(x_{1}\right.$, & $x_{2}$, & $\ldots$, & $x_{s}$, & $x_{s+1}$, & $\ldots$, & $\left.x_{n}\right)$ & $Q_{1}$ & $Q_{2}$ & $Q_{7}$ & $Q_{8}$ & $Q_{6}$ & $Q_{5}$ \\
\hline \hline$(0$, & 0, & $\ldots$, & 0, & 0, & $\ldots$, & $0)$ & 000 & 000 & 000 & 000 & 000 & 000 \\
$(1$, & 0, & $\ldots$, & 0, & 0, & $\ldots$, & $0)$ & 000 & 000 & 011 & 110 & 000 & 000 \\
$(1$, & 1, & $\ldots$, & 1, & 0, & $\ldots$, & $0)$ & 101 & 101 & 110 & 011 & 101 & 101 \\
$(1$, & 1, & $\ldots$, & 1, & 1, & $\ldots$, & $1)$ & 110 & 011 & 110 & 011 & 011 & 110 \\
\hline
\end{tabular}

Как видно из таблицы (с учетом леммы 7), все константные неисправности элементов цепочки $C$ обнаруживаются на множестве $\hat{T}_{n}$ и второе утверждение теоремы выполняется для всех этих элементов.

Таким образом, построенная в подслучае 1.2 .1 схема $S_{B^{\prime}}^{f}$ является неизбыточной и указанные 4 набора образуют единичный проверяющий тест для нее.

Подслучай 1.2.2. Пусть функция $f$ такова, что в $P_{f}$ есть линейные слагаемые, входящие в качестве множителей-переменных в $K_{1}$. Будем, не ограничивая общности, считать, что переменные $x_{1}, x_{2}, \ldots, x_{k}$ из $K_{1}$ входят в $P_{f}$ в качестве слагаемых $K_{t-k+1}, K_{t-k+2}, \ldots, K_{t}$ (соответственно; здесь $1 \leqslant k \leqslant s$ ). Все переменные, не входящие в $K_{1}$ в качестве множителей, но входящие в $P_{f}$ в качестве слагаемых, образуют слагаемые $K_{q+1}, K_{q+2}, \ldots, K_{t-k}$.

Неизбыточную схему $S_{B^{\prime}}^{f}$, реализующую $f$ и допускающую единичный проверяющий тест длины 4 относительно константных неисправностей на выходах элементов, будем строить в полной аналогии с подслучаем 1.2.1, незначительные уточнения коснутся лишь последовательности элементов $D_{3}$ цепи $C$ блока $S_{4}$. Именно, на правые входы первых $t-k-q$ элементов $D_{3}$ подаются переменные, представляющие собой слагаемые $K_{q+1}, K_{q+2}, \ldots, K_{t-k}$ и не встречающиеся в $K_{1}$ (как и в случае $1.2 .1,-$ за исключением того, что там последний элемент этой цепочки мог быть элементом эквивалентности); обозначим последовательность из этих элементов через $D_{3}^{\prime}$. На правые входы остальных $k$ элементов $D_{3}$ подаются слагаемые $K_{t-k+1}=x_{1}, K_{t-k+2}$, $\ldots, K_{t}$ (в указанном порядке), представляющие собой переменные $x_{1}, x_{2}, \ldots, x_{k}$, встречающиеся в $K_{1}$; обозначим последовательность из этих элементов через $D_{3}^{\prime \prime}$. Каждая из цепей $D_{2}, D_{3}^{\prime}$ может не содержать элементов. 
Из анализа таблиц, построенных в подслучаях 1.2.1.1 и 1.2.1.2, следует, что значения на выходе элемента цепочки $C$, непосредственно предшествующего первому элементу цепочки $D_{3}^{\prime \prime}$, могут образовывать либо столбец $(0,0,1,0)^{T}$, либо столбец $(0,0,1,1)^{T}$. Достроим эти таблицы для не рассмотренных ранее элементов цепи $D_{3}^{\prime \prime}$.

Подслучай 1.2.2.1. Значения на выходе элемента цепочки $C$, непосредственно предшествующего первому элементу цепочки $D_{3}^{\prime \prime}$, образуют столбец $(0,0,1,0)^{T}$. Столбец $Q_{9}$ соответствует первому элементу цепочки $D_{3}^{\prime \prime}$, столбец $Q_{10}$ соответствует элементам цепочки $D_{3}^{\prime \prime}$ с четными номерами, столбец $Q_{11}$ соответствует элементам цепочки $D_{3}^{\prime \prime}$ с нечетными большими одного номерами:

\begin{tabular}{|ccccccc||c|c|c|}
\hline$\left(x_{1}\right.$, & $x_{2}$, & $\ldots$, & $x_{s}$, & $x_{s+1}$, & $\ldots$, & $\left.x_{n}\right)$ & $Q_{9}$ & $Q_{10}$ & $Q_{11}$ \\
\hline \hline$(0$, & 0, & $\ldots$, & 0, & 0, & $\ldots$, & $0)$ & 000 & 000 & 000 \\
$(1$, & 0, & $\ldots$, & 0, & 0, & $\ldots$, & $0)$ & 011 & 101 & 101 \\
$(1$, & 1, & $\ldots$, & 1, & 0, & $\ldots$, & $0)$ & 110 & 011 & 110 \\
$(1$, & 1, & $\ldots$, & 1, & 1, & $\ldots$, & $1)$ & 011 & 110 & 011 \\
\hline
\end{tabular}

Как видно из таблицы (с учетом леммы 7), все константные неисправности элементов цепочки $D_{3}^{\prime \prime}$ обнаруживаются на множестве $\hat{T}_{n}$, и второе утверждение теоремы выполняется для всех этих элементов.

Подслучай 1.2.2.2. Значения на выходе элемента цепочки $C$, непосредственно предшествующего первому элементу цепочки $D_{3}^{\prime \prime}$, образуют столбец $(0,0,1,1)^{T}$. Столбец $Q_{12}$ соответствует первому элементу цепочки $D_{3}^{\prime \prime}$, столбец $Q_{13}$ соответствует элементам цепочки $D_{3}^{\prime \prime}$ с четными номерами, столбец $Q_{14}$ соответствует элементам цепочки $D_{3}^{\prime \prime}$ с нечетными большими одного номерами:

\begin{tabular}{|ccccccc||c|c|c|}
\hline$\left(x_{1}\right.$, & $x_{2}$, & $\ldots$, & $x_{s}$, & $x_{s+1}$, & $\ldots$, & $\left.x_{n}\right)$ & $Q_{12}$ & $Q_{13}$ & $Q_{14}$ \\
\hline \hline$(0$, & 0, & $\ldots$, & 0, & 0, & $\ldots$, & $0)$ & 000 & 000 & 000 \\
$(1$, & 0, & $\ldots$, & 0, & 0, & $\ldots$, & $0)$ & 011 & 101 & 101 \\
$(1$, & 1, & $\ldots$, & 1, & 0, & $\ldots$, & $0)$ & 110 & 011 & 110 \\
$(1$, & 1, & $\ldots$, & 1, & 1, & $\ldots$, & $1)$ & 110 & 011 & 110 \\
\hline
\end{tabular}

Как видно из таблицы (с учетом леммы 7), все константные неисправности элементов цепочки $D_{3}^{\prime \prime}$ обнаруживаются на множестве $\hat{T}_{n}$, и второе утверждение теоремы выполняется для всех этих элементов.

Значит, и в подслучае 1.2.2 наборы $\left(\tilde{0}^{n}\right),\left(\tilde{1}^{n}\right),\left(1, \tilde{0}^{n-1}\right),\left(\tilde{1}^{s}, \tilde{0}^{n-s}\right)$ образуют единичный проверяющий тест для схемы $S_{B^{\prime}}^{f}$.

Случай 2. Пусть функция $f\left(\tilde{x}^{n}\right)-$ линейная.

Подслучай 2.1. Функция $f\left(\tilde{x}^{n}\right)$ - константа 0 (соответственно, константа 1 ). Тогда реализующая ее схема $S_{B^{\prime}}^{f}$ моделирует формулу $x_{1} \oplus x_{1}$ (соответственно, $x_{1} \sim$ $\left.x_{1}\right)$. В этих случаях набор $\left(\tilde{0}^{n}\right)$ образует единичный проверяющий тест.

Подслучай 2.2. Функция $f\left(\tilde{x}^{n}\right)$ - переменная (например, $x_{1}$ ). Тогда схема $S_{B^{\prime}}^{f}$ не содержит элементов и состоит только из входной вершины $x_{1}$, объявленной также и выходной вершиной. В этом случае схема абсолютно надежна, тестировать ничего не надо.

Подслучай 2.3. Функция $f\left(\tilde{x}^{n}\right)$ - отрицание переменной (например, $\left.\bar{x}_{1}\right)$. Тогда схема $S_{B^{\prime}}^{f}$ состоит из двух элементов и моделирует формулу $\left(x_{1} \oplus\left(x_{1} \sim x_{1}\right)\right)$. В этом случае наборы $\left(\tilde{0}^{n}\right),\left(\tilde{1}^{n}\right)$ образуют единичный проверяющий тест. 
Подслучай 2.4. Функция $f\left(\tilde{x}^{n}\right)$ имеет хотя бы две существенные переменные. Будем, не ограничивая общности, считать, что $f\left(\tilde{x}^{n}\right)=x_{1} \oplus x_{2} \oplus \cdots \oplus x_{n} \oplus a_{0}\left(a_{0} \in\right.$ $\{0,1\})$. Тогда схема $S_{B^{\prime}}^{f}$ состоит из цепочки $C$ элементов сумм по модулю 2 и, если $a_{0}=1$, одного (выходного) элемента $E_{\sim}$ типа „эквивалентность“. На входы цепочки подаются переменные $x_{1}, x_{2}, \ldots, x_{n}$ (в указанном порядке). Построим таблицу значений на входах и выходах этих элементов на наборах $\left(\tilde{0}^{n}\right),\left(1, \tilde{0}^{n-1}\right)$ и $\left(\tilde{1}^{n}\right)$. Столбец $Q_{15}$ соответствует первому элементу цепочки $C$, столбец $Q_{16}$ соответствует элементам цепочки $C$ с четными номерами, столбец $Q_{17}$ соответствует элементам цепочки $C$ с нечетными большими одного номерами:

\begin{tabular}{|cccc||c|c|c|}
\hline$\left(x_{1}\right.$, & $x_{2}$, & $\ldots$, & $\left.x_{n}\right)$ & $Q_{15}$ & $Q_{16}$ & $Q_{17}$ \\
\hline \hline$(0$, & 0, & $\ldots$, & $0)$ & 000 & 000 & 000 \\
$(1$, & 0, & $\ldots$, & $0)$ & 101 & 101 & 101 \\
$(1$, & 1, & $\ldots$, & $1)$ & 110 & 011 & 110 \\
\hline
\end{tabular}

Как видно из таблицы (с учетом леммы 7), все константные неисправности элементов цепочки $D_{3}^{\prime \prime}$ обнаруживаются на множестве $\hat{T}_{n}$ и второе утверждение теоремы выполняется для всех этих элементов.

Значит, в подслучае 2.4 наборы $\left(\tilde{0}^{n}\right),\left(\tilde{1}^{n}\right),\left(1, \tilde{0}^{n-1}\right)$ образуют единичный проверяющий тест для схемы $S_{B^{\prime}}^{f}$.

Теорема доказана.

Теорема 2. Для каждой булевой функции $f\left(\tilde{x}^{n}\right)$ справедливы следующие утверждения:

1) существует реализующая функиию $f\left(\tilde{x}^{n}\right)$ неизбыточная $C Ф \vartheta S_{B^{\prime \prime}}^{f}$ в базисе $B^{\prime \prime}=\{\bar{x}, x \& \bar{y}, x \oplus y, x \sim y\}$, допускающая некоторый единичный проверяющий тест $\check{T}_{n}$ длины, не превосходящей четырех, относительно произвольных константных неисправностей на выходах элементов;

2) при этом, если число существенных переменных функиии $f$ не менъше двух, то для каждого функиионального элемента $E$ суммы по модулю 2 или эквивалентности в схеме $S_{B^{\prime \prime}}^{f}$ найдутся три набора из множества $\check{T}_{n}$, на каждом из которых обнаруживается константная неисправность на выходе E, и при их подаче на входы схемы на входах элемента $Е$ возникают три различных набора.

Доказательство. аналогично доказательству теоремы 1 . Обозначим $g(x, y)=x \bar{y}$.

Пусть $f\left(\tilde{x}^{n}\right)$ - произвольная булева функция, зависящая (существенно или фиктивно) от переменных $x_{1}, x_{2}, \ldots, x_{n}$.

Случай 1. Пусть функция $f-$ нелинейная.

Подслучай 1.1. Пусть функция $f$ существенно зависит ровно от двух переменных.

Положим $\check{T}_{2}=\{(0,1),(1,1),(1,0)\}$.

Для функции $f=x_{1} \bar{x}_{2}$ искомая схема состоит из одного элемента $g\left(x_{1}, x_{2}\right)$. Для функции $f=x_{1} x_{2}$ искомая схема $S_{B^{\prime \prime}}^{f}$ моделирует формулу $g\left(x_{1}, \bar{x}_{2}\right)$. Для функций $x_{1} \downarrow x_{2}, x_{1} \vee x_{2}, \bar{x}_{1} \vee x_{2}, x_{1} \mid x_{2}$ искомые схемы моделируют формулы $g\left(\bar{x}_{1}, x_{2}\right), \bar{g}\left(\bar{x}_{1}, x_{2}\right)$, $\bar{g}\left(x_{1}, x_{2}\right), \bar{g}\left(x_{1}, \bar{x}_{2}\right)$ соответственно. Легко показать, что для каждой из этих схем множество $\check{T}_{2}$ образует единичный проверяющий тест.

Подслучай 1.2. Пусть функция $f$ существенно зависит не менее чем от трех переменных. Положим $\check{f}\left(x_{1}, x_{2}, \ldots, x_{n}\right)=f\left(x_{1}, \bar{x}_{2}, \ldots, \bar{x}_{n}\right)$. Перепишем полином 
Жегалкина функции $\check{f}$ в виде $P_{\check{f}}=K_{1} \oplus K_{2} \oplus \cdots \oplus K_{t} \oplus a_{0}$, где $K_{i}$ - конъюнкции различных переменных или переменные $(i=\overline{1, t}), a_{0} \in\{0,1\}$. Пусть, далее, $K_{1}$ - слагаемое минимального ранга в полиноме Жегалкина функции $\check{f}$, отличное от константы и переменной. Будем, не ограничивая общности, считать, что $K_{1}=x_{1} x_{2} \cdots x_{s}(2 \leqslant s \leqslant n)$, и что слагаемые $K_{1}, K_{2}, \ldots, K_{q}(1 \leqslant q \leqslant t)-$ нелинейные, а слагаемые $K_{q+1}, K_{q+2}, \ldots, K_{t}$ - линейные (точнее, переменные).

Схема $S_{B^{\prime \prime}}^{f}$ составлена из блоков $S_{1}, S_{2}, S_{3}, S_{4}$, как показано на рисунке 7.

Для ее описания используем схему $S_{B^{\prime}}^{\check{f}}$, которая реализует $\check{f}$ и строится в соответствии с доказательством подслучая 1.2 теоремы 1.

Блок $S_{1}$ схемы $S_{B^{\prime \prime}}^{f}$ получается из блока $S_{1}$ схемы $S_{B^{\prime}}^{\check{f}}$ следующим образом. Рассмотрим произвольный вход произвольного конъюнктора блока $S_{1}$ схемы $S_{B^{\prime}}^{\check{f}}$, на который подается входная переменная $x_{j}$, отличная от $x_{1}$. Построим для этого входа индивидуальный инвертор, его выход подадим на рассматриваемый вход конъюнктора (отсоединив от этого входа $x_{j}$ ), а на вход этого инвертора подадим переменную $x_{j}$. Пусть такая операция проделана со всеми входами конъюнкторов указанного выше вида. Заметим: на правый вход каждого конъюнктора подается неветвящийся выход инвертора, на вход которого подается переменная $x_{j}$, отличная от $x_{1}$. (Возможно, что на левые входы некоторых бывших первых конъюнкторов цепочек блока $S_{1}$ схемы $S_{B^{\prime}}^{\check{f}}$ теперь тоже подаются выходы инверторов.) Заменим теперь каждый инвертор, выход которого подается на правый вход конъюнктора, на эквивалентный этой подсхеме функциональный элемент $g$ (эквивалентная подстановка). В результате каждая цепочка конъюнкторов $C_{i}$ блока $S_{1}$ схемы $S_{B^{\prime}}^{\check{f}}$ превратилась в цепочку $C_{i}$ элементов отрицания и элементов $g$ (в блоке $S_{1}$ схемы $S_{B^{\prime \prime}}^{f}$ ), в которой не более одного элемента отрицания и, если таковой имеется, то он является первым элементом цепочки $(i=\overline{1, q})$.

Блок $S_{2}$ схемы $S_{B^{\prime \prime}}^{f}$ представляет собой копию блока $S_{1}$ схемы $S_{B^{\prime \prime}}^{f}$, в которой удалены последние элементы в каждой из цепочек $C_{1}, C_{2}, \ldots, C_{q}$. Пусть число элементов в $S_{2}$ есть $L_{2}$, обозначим элементы из $S_{2}$ через $E_{1}^{0}, E_{2}^{0}, \ldots, E_{L_{2}}^{0}$, считая, что в начале этого перечня следуют элементы из копии цепочки $C_{1}$ в соответствии с их порядком в цепочке, затем идут элементы из копии цепочки $C_{2}$ в соответствии с их порядком в цепочке, и так далее, - завершают список элементы из копии цепочки $C_{q}$ в соответствии с их порядком в цепочке. Отметим, что блок $S_{2}$ может не содержать элементов.

Блок $S_{3}$ состоит из трехвходовых подсхем $\Sigma_{i}^{3}\left(i=\overline{1, L_{2}}\right)$, состоящих из функциональных элементов базиса $B^{\prime \prime}$ (при $L_{2}=0$ блок $S_{3}$ не содержит элементов). Для каждой пары „дублирующих друг друга“ элементов из блоков $S_{1}$ и $S_{2}$ строится одна такая подсхема. Для каждого элемента $E^{\prime \prime}$ из блока $S_{2}$, являющегося выходным элементом цепочки из блока $S_{2}$, и его копии $E^{\prime}$ в блоке $S_{1}$ соответствующая подсхема $\Sigma_{i}^{3}$ представляет собой изображенную на рисунке 3 трехвходовую схему $\check{S}_{B^{\prime \prime}}^{\psi}$, реализующую функцию $\psi(x, y, z)$; на левый вход схемы $\check{S}_{B^{\prime \prime}}^{\psi}$ подается выход элемента $E^{\prime}$, на средний вход - выход элемента $E^{\prime \prime}$, а на правый - переменная $x_{1}$. Для каждого элемента $E^{\prime \prime}$ из блока $S_{2}$, отличного от выходного элемента цепочки из блока $S_{2}$, и его копии $E^{\prime}$ в блоке $S_{1}$ соответствующая подсхема $\Sigma_{i}^{3}$ представляет собой трехвходовую схему $\check{S}_{B^{\prime \prime}}^{\psi^{\prime}}$, изображенную на рисунке 4 ; на левый вход этой подсхемы подается выход элемента $E^{\prime}$, на средний вход $\Sigma_{i}^{3}$ - выход элемента $E^{\prime \prime}$, а на правый - переменная $x_{1}$. Заметим, что на выходе каждой подсхемы $\Sigma_{i}^{3}$ при отсутствии неисправностей реализуется функция $x_{1}$. 
Блок $S_{4}$ состоит из цепочки $C$ элементов сумм по модулю 2 и элементов типа „эквивалентность“. Пусть $a_{0}=0$. На левый вход самого первого элемента цепочки $C$ подается выход цепочки $C_{1}$ блока $S_{1}$; на левый вход каждого элемента цепочки $C$, начиная со второго, подается выход предыдущего элемента этой цепочки; на правые входы первых $(q-1)$ элементов цепочки $C$ подаются выходы цепочек $C_{2}, \ldots$, $C_{q}$ блока $S_{1}$ (в указанном порядке), эти элементы цепочки $C$ являются элементами сумм по модулю 2 ; на правые входы следующих $L_{2}$ элементов цепочки $C$ подаются выходы всех трехвходовых подсхем $\Sigma_{1}^{3}, \Sigma_{2}^{3}, \ldots, \Sigma_{L_{2}}^{3}$ из блока $S_{3}$ в указанном порядке (и, если $L_{2}$ нечетное, то на правый вход следующего элемента цепочки $C$ подается переменная $x_{1}$ ), эти элементы цепочки $C$ также являются элементами сумм по модулю 2 ; на правые входы следующих $t-q$ элементов цепочки $C$ подаются переменные, являющиеся линейными слагаемыми в $P_{\breve{f}}$, в таком порядке: сначала линейные слагаемые, не входящие в конъюнкцию $K_{1}$ в $P_{\check{f}}$ (если таковые имеются в $\left.P_{\check{f}}\right),-$ в порядке возрастания индексов этих переменных, затем линейные слагаемые, входящие в конъюнкцию $K_{1}$ в $P_{\check{f}}$ (если таковые имеются в $\left.P_{\check{f}}\right),-$ в порядке возрастания индексов этих переменных (при этом все указанные элементы цепочки $C$, на правые входы которых не подается $x_{1}$, являются элементами сумм по модулю 2 ). При $a_{0}=1$ отличие заключается лишь в том, что последний элемент в цепочке $C$ при $a_{0}=0$ должен быть заменен на „противоположный“ („эквивалентность“ надо заменить на сумму по модулю 2 или наоборот). Обозначим через $D_{1}$ последовательность всех элементов цепочки $C$ (в порядке следования в цепочке), у которых хотя бы на один вход подается выход цепочки из блока $S_{1}$. Пусть, далее, $D_{2}-$ последовательность всех элементов цепочки $C$ (в порядке следования в цепочке), у которых на правый вход подается выход элемента из блока $S_{3}$ (если $L_{2}$ нечетное, то к $D_{2}$ относится и следующий функциональный элемент суммы по модулю 2 , на вход которого подается переменная $x_{1}$; таким образом, в $D_{2}$ четное число элементов). Наконец, пусть $D_{3}$ - последовательность всех элементов цепочки $C$ (в порядке следования в цепочке), у которых на правый вход подается входная переменная. Заметим, что при $q=1$ у $D_{1}$ и $D_{2}$ есть один общий элемент, а в остальных случаях попарно общих элементов у $D_{1}, D_{2}$ и $D_{3}$ нет; последовательности $D_{2}$ и $D_{3}$ могут не содержать элементов. Отметим, что цепочка $C$ содержит хотя бы один элемент.

Выход последнего элемента цепочки $C$ является выходом схемы $S_{B^{\prime \prime}}^{f}$.

Легко видеть, что при отсутствии неисправностей схема $S_{B^{\prime}}^{f}$ реализует функцию $f$ (поскольку реализуемая на выходе функция, очевидно, вычисляется так: $\left.\check{f}\left(x_{1}, \bar{x}_{2}, \ldots, \bar{x}_{n}\right)=f\left(x_{1}, x_{2}, \ldots, x_{n}\right)\right)$.

Положим $\check{T}_{n}=\left\{\left(0, \tilde{1}^{n-1}\right),\left(1, \tilde{0}^{n-1}\right),\left(\tilde{1}^{n}\right),\left(1, \tilde{0}^{s-1}, \tilde{1}^{n-s}\right)\right\}$. Оказывается, $\check{T}_{n}-$ единичный проверяющий тест для схемы $S_{B^{\prime \prime}}^{f}$. (Это, фактически, следует из того, что наборы теста $\check{T}_{n}$ получаются из соответствующих наборов теста $\hat{T}_{n}$ из подслучая 1.2 доказательства теоремы 1 инвертированием значений переменных $x_{2}, \ldots, x_{n}$.)

На наборе $\left(0, \tilde{1}^{n-1}\right)$ на выходах всех элементов из блоков $S_{1}, S_{2}$ будут нули, на входах каждой подсхемы $\Sigma_{i}^{3}\left(i=\overline{1, L_{2}}\right)$ возникнут наборы $(0,0,0)$. На наборе $\left(1, \tilde{0}^{n-1}\right)$ на выходах всех элементов из блоков $S_{1}, S_{2}$ будут единицы, на входах каждой подсхемы $\Sigma_{i}^{3}$ возникнут наборы $(1,1,1)$. На наборе $\left(\tilde{1}^{n}\right)$ на выходах всех элементов из блоков $S_{1}, S_{2}$ будут нули, на входах каждой подсхемы $\Sigma_{i}^{3}$ возникнут наборы $(0,0,1)$. Множество $\{(0,0,0),(1,1,1),(0,0,1)\}$ образует единичный проверяющий тест для каждой из схем $\check{S}_{B^{\prime \prime}}^{\psi}, \check{S}_{B^{\prime \prime}}^{\psi^{\prime}}$ по леммам 10 и 11. В силу устройства схем $S_{B^{\prime \prime}}^{f}$, $\check{S}_{B^{\prime \prime}}^{\psi}, \check{S}_{B^{\prime \prime}}^{\psi^{\prime}}$ каждый элемент суммы по модулю 2 и эквивалентности в схеме $S_{B^{\prime \prime}}^{f}$ имеет неветвящийся выход и лежит на продолжающейся до выхода схемы $S_{B^{\prime \prime}}^{f}$ цепи, 
состоящей только из элементов суммы по модулю 2 и эквивалентности. Значит, в силу леммы 7 любая константная неисправность выходного элемента (а это элемент суммы по модулю 2) любой подсхемы $\Sigma_{i}^{3}$ будет обнаружена на множестве входных наборов $\check{T}_{n}$ схемы $S_{B^{\prime \prime}}^{f}$. Поэтому по леммам $2,7,8$ и 9 на наборах из $\check{T}_{n}$ будут обнаруживаться все константные неисправности всех элементов, лежащих в подсхемах $\Sigma_{i}^{3}$ схемы $S_{B^{\prime \prime}}^{f}$. Заметим, что для всех элементов суммы по модулю 2 и эквивалентности, лежащих в подсхемах $\Sigma_{i}^{3}$ схемы $S_{B^{\prime \prime}}^{f}$, второе утверждение настоящей теоремы выполняется, как это непосредственно следует из анализа таблиц из доказательств лемм 10-11 и из леммы 7.

Легко видеть, что на наборе $\left(0, \tilde{1}^{n-1}\right)$ обнаруживаются все неисправности типа „константа 1“ на выходах всех элементов из блоков $S_{1}, S_{2}$. Действительно, $f\left(\widetilde{0}^{n}\right)=a_{0}$, и на выходах всех элементов из блоков $S_{1}, S_{2}$ (при отсутствии в схеме неисправностей) на данном наборе оказываются нули. Если неисправность типа „1“ произошла на выходе элемента $E^{\prime}$ блока $S_{1}$, не являющегося последним элементом одной из цепочек $C_{1}, C_{2}, \ldots, C_{q}$, то на выходе следующего за $E^{\prime}$ элемента в цепочке будет по-прежнему 0, и на выходе цепочки, в которую входит $E^{\prime}$, будет по-прежнему 0. Зато на выходе трехвходовой подсхемы $\Sigma_{i}^{3}$, на вход которой подается выход $E^{\prime}$, появится 1 , и на выходе схемы $S_{B^{\prime \prime}}^{f}$ значение изменится на неправиль-

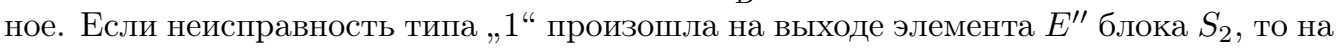
выходе следующих за $E^{\prime \prime}$ элементов (если они есть) в цепочке будут по-прежнему 0. Зато на выходе трехвходовой подсхемы $\Sigma_{i}^{3}$, на вход которой подается выход $E^{\prime \prime}$, появится 1 , и на выходе схемы $S_{B^{\prime \prime}}^{f}$ значение изменится на неправильное. Если же неисправность типа „1“ произошла на выходе элемента $E^{\prime}$ блока $S_{1}$, являющегося последним элементом одной из цепочек $C_{1}, C_{2}, \ldots, C_{q}$, то, в силу того, что выход этого элемента не ветвится и подается на вход идущей до выхода схемы цепочки элементов сумм по модулю 2 и эквивалентности, значение на выходе схемы $S_{B^{\prime \prime}}^{f}$ изменится на неправильное.

На наборе $\left(1, \tilde{0}^{n-1}\right)$ обнаруживаются все неисправности типа „0“ на выходах всех элементов из блоков $S_{1}, S_{2}$. Действительно, на этом наборе на выходах всех элементов из блоков $S_{1}, S_{2}$ и трехвходовых подсхем $\Sigma_{i}^{3}\left(i \in\left\{1, \ldots, L_{2}\right\}\right)$ схемы $S_{B^{\prime \prime}}^{f}$ (при отсутствии в ней неисправностей) оказываются единицы. Неисправность типа „0“ на выходе элемента $E^{\prime}$ блока $S_{1}$, не являющегося последним элементом цепочки $C_{i}$ $(i \in\{1,2, \ldots, q\})$, будет обнаружена на данном наборе, поскольку значение на выходе цепочки элементов, в которую входит $E^{\prime}$ (и только этой цепочки), изменится с 1 на 0 , а значения на выходах всех трехвходовых подсхем $\Sigma_{i}^{3}$ останутся равными 1. Неисправность типа „0“ на выходе элемента $E^{\prime \prime}$ блока $S_{2}$ будет обнаружена на наборе $\left(1, \tilde{0}^{n-1}\right)$, поскольку значение на выходе трехвходовой подсхемы $\Sigma_{i}^{3}$, на средний вход которой подается выход последнего элемента из той цепочки блока $S_{2}$, которой принадлежит $E^{\prime \prime}$, изменится с 1 на 0 , а значения на выходах остальных трехвходовых подсхем $\Sigma_{j}^{3}$ останутся равными 1 . Если же неисправность типа „0“ произошла на выходе последнего элемента цепочки $C_{i}$, то, в силу того, что выход этого элемента не ветвится и подается на вход идущей до выхода схемы цепочки элементов сумм по модулю 2 и эквивалентности, значение на выходе схемы $S_{B^{\prime \prime}}^{f}$ на этом наборе изменится.

На наборе $\left(\tilde{1}^{n}\right)$ на выходах всех элементов из блоков $S_{1}, S_{2}$ схемы $S_{B^{\prime}}^{f}$ (при отсутствии неисправностей) оказываются нули, на выходах всех трехвходовых подсхем $\Sigma_{i}^{3}$ - единицы. 
На наборе $\left(1, \tilde{0}^{s-1}, \tilde{1}^{n-s}\right)$ (при отсутствии в схеме неисправностей) на выходах всех элементов цепочки $C_{1}$ блока $S_{1}$ и на выходах копий этих конъюнкторов в блоке $S_{2}$ оказываются единицы, на выходах всех остальных элементов из блоков $S_{1}, S_{2}$ нули, на выходах всех трехвходовых подсхем $\Sigma_{i}^{3}$ оказываются единицы (поскольку на правые входы трехвходовых подсхем $\Sigma_{i}^{3}$ подается переменная $x_{1}$, принимающая на данном наборе значение 1).

Чтобы понять, какие неисправности элементов цепочки $C$ обнаруживаются на наборах множества $\check{T}_{n}$, сравним эту цепочку с цепочкой $C$ схемы $S_{B^{\prime}}^{\check{f}}$. Если элемент $E$ цепочки $C$ схемы $S_{B^{\prime \prime}}^{f}$ является элементом в цепочке $D_{1}\left(D_{3}\right)$, то сопоставим ему элемент $\check{E}$ с тем же номером в цепочке $D_{1}$ (соответственно $D_{3}$ ) схемы $S_{B^{\prime}}^{\check{f}}$, 一 ясно, что длины цепочек $D_{1}\left(D_{3}\right)$ в указанных схемах совпадают. Может, однако, оказаться, что в схеме $S_{B^{\prime}}^{\check{f}}$ цепочка $D_{2}$ не содержит элементов, а в схеме $S_{B^{\prime \prime}}^{f}$ цепочка $D_{2}$ содержит элементы (противоположная ситуация невозможна по построению схемы $\left.S_{B^{\prime \prime}}^{f}\right)$, - тогда следует удлинить в схеме $S_{B^{\prime}}^{\check{f}}$ цепочку $C$ на два элемента суммы по модулю 2 , располагающихся между цепочками $D_{1}$ и $D_{3}$, на правые входы каждого из которых подается $x_{1}$, а на левые - выход предыдущего элемента цепочки, и заметить, что таблицы значений на входах и выходах элементов цепочки $C$ на наборах $\hat{T}_{n}$ фактически описаны и для этой ситуации в доказательстве подслучая 1.2 теоремы 1. Пусть элемент $E$ цепочки $C$ схемы $S_{B^{\prime \prime}}^{f}$ является элементом в цепочке $D_{2}$, тогда сопоставим ему элемент $\check{E}$ с номером той же четности в цепочке $D_{2}$ схемы $S_{B^{\prime}}^{\check{f}}$ (возможно, дополненной двумя элементами, как описано выше). Остается заметить, что по построению схемы $S_{B^{\prime \prime}}^{f}$ для каждого элемента $E$ ее цепочки $C$ таблица значений на входах и выходах элемента $E$ на наборах $\left(0, \tilde{1}^{n-1}\right),\left(1, \tilde{0}^{n-1}\right),\left(\tilde{1}^{n}\right),\left(1, \tilde{0}^{s-1}, \tilde{1}^{n-s}\right)$ либо совпадает с таблицей значений на входах и выходах сопоставленного элементу $E$ элемента $\check{E}$ схемы $S_{B^{\prime}}^{\check{f}}$ на наборах $\left(\tilde{0}^{n}\right),\left(\tilde{1}^{n}\right),\left(1, \tilde{0}^{n-1}\right),\left(\tilde{1}^{s}, \tilde{0}^{n-s}\right)$ (соответственно), либо получается из последней инвертированием некоторых столбцов, что с учетом доказательства подслучая 1.2 теоремы 1 приводит к тому, что единичные неисправности всех элементов цепочки $C$ схемы $S_{B^{\prime \prime}}^{f}$ обнаруживаются на наборах множества $\check{T}_{n}$, и вторая часть утверждения теоремы для этих элементов выполнена.

Теорема для данного подслучая доказана.

Случай 2. Пусть функция $f\left(\tilde{x}^{n}\right)$ - линейная. В этом случае доказательство повторяет доказательство случая 2 теоремы 1.

Теорема доказана.

\section{Синтез легкотестируемых неизбыточных схем в бази- $\operatorname{cax} B_{1}=\{\bar{x}, x \& y\}, B_{2}=\{\bar{x}, x \vee y\}, B_{3}=\{x \mid y\}, B_{4}=\{x \downarrow y\}$}

Теорема 3. Для каждой отличной от константы булевой функции $f$ существует реализующая ее неизбыточная $C$ Э $S_{B_{1}}^{f}$ в базисе $B_{1}=\{\bar{x}, x \& y\} \quad\left(C \Phi \vartheta S_{B_{2}}^{f}\right.$ в базисе $\left.B_{2}=\{\bar{x}, x \vee y\}\right)$, допускающая единичный проверяющий тест длины, не превосходящей четырех, относительно инверсных и произвольных константных неисправностей на выходах элементов.

Доказательство. Рассмотрим базис $B_{1}$. Для функции $f=\bar{x}_{1}$ искомая СФЭ моделирует формулу $\bar{x}_{1}$. Для функции $f=x_{1}$ искомая СФЭ не содержит элементов. Пусть теперь функция $f$ существенно зависит не менее чем от двух переменных. 
Воспользуемся схемой $S_{B^{\prime}}^{f}$, построенной в теореме 1 . Пусть $E-$ какой-то элемент суммы по модулю 2 или эквивалентности в этой схеме. Пусть на входных наборах из $\hat{T}_{n}$ (из теоремы 1 ) на входах этого элемента возникает тройка различных наборов $A$ из множества $\{(0,0),(0,1),(1,0),(1,1)\}$ (такая тройка найдется по части 2 теоремы 1$)$, и на каждом наборе из $A$ будет обнаруживаться какая-то константная неисправность на выходе $E$. Тогда по лемме 13 найдется эквивалентная элементу $E$ неизбыточная схема $S$ в базисе $B_{1}$, для которой множество $A$ будет единичным проверяющим тестом. Осуществим эквивалентную подстановку схемы $S$ вместо элемента $E$. Выполним аналогичную процедуру для каждого элемента суммы по модулю 2 или эквивалентности в схеме $S_{B^{\prime}}^{f}$. По лемме 2 полученная схема $S_{B_{1}}^{f}$ в базисе $B_{1}$ неизбыточна, а множество $\hat{T}_{n}$ образует для нее единичный проверяющий тест.

Чтобы доказать теорему для базиса $B_{2}$, надо воспользоваться принципом двойственности: построить схему $S_{B_{1}}^{f^{*}}$ в базисе $B_{1}$ для двойственной к $f$ функции и заменить все функциональные элементы на двойственные. Теорема доказана.

Теорема 4. Для каждой отличной от константы булевой функиии $f$ существует реализующая ее неизбыточная $C$ Э $S_{B}^{f}$ в базисе $B \in\left\{B_{3}, B_{4}\right\}$ (где $B_{3}=\{x \mid y\}$, $\left.B_{4}=\{x \downarrow y\}\right)$, допускающая единичный проверяющий тест длины, не превосходящей четырех, относительно инверсных и произвольных константных неисправностей на выходах элементов.

Доказательство. Рассмотрим базис $B_{3}$. В схеме $S_{B_{1}}^{f}$ достаточно заменить по лемме 3 конъюнкторы на цепочки из конъюнктора, инвертора и инвертора (следующих в указанном порядке), а затем каждую цепочку из конъюнктора и следующего за ним инвертора заменить на эквивалентный двухвходовый элемент базиса $B_{3}$. Вместо каждого из оставшихся инверторов следует эквивалентно подставить одновходовую подсхему, вход которой подается на оба входа единственного (в подсхеме) элемента штриха Шеффера, чтобы получить искомую схему $S_{B_{3}}^{f}$. Случай базиса $B_{4}$ - двойственный. Теорема доказана.

Теорема 5. Для каждой равной константе булевой функиии $f$ не существует

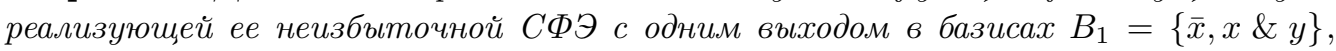
$B_{2}=\{\bar{x}, x \vee y\}$.

Доказательство проведем для базиса $B_{1}$ (для $B_{2}$ - двойственным образом). Пусть $S$ - минимальная неизбыточная СФЭ в базисе $B_{1}$, реализующая константу. Тогда ее выходной элемент - не инвертор, а конъюнктор. Существует цепь (хотя бы одна), ведущая от какого-то входа к выходу схемы $S$ и такая, что в ней имеются элементы $E$ и $E^{\prime}$, удовлетворяющие следующим условиям: на выходе элемента $E$ реализуется константа и хотя бы на один из его входов подается выход элемента $E^{\prime}$, причем на выходе элемента $E^{\prime}$ реализуется функция, отличная от константы (существование следует из того, что на входах схемы реализуются отличные от констант функции, а на выходе - константа, и того, что конъюнкция переменных и отрицание переменной отличны от констант). Выберем такую обладающую этими свойствами пару элементов $\left(E, E^{\prime}\right)$, в которой глубина элемента $E^{\prime}$ (то есть максимум длины ориентированного пути до элемента $E^{\prime}$ в схеме) максимальна. Ясно, что элемент $E-$ не инвертор. Значит, это - конъюнктор. Тогда реализуемая на его выходе константа - 0 (так как иначе на входах $E$ реализовывались бы константы 1$)$. Пусть выходы элемента $E^{\prime}$ подаются на входы элементов $E, E_{1}, \ldots, E_{r}$. Тогда, в силу максимальности глубины $E^{\prime}$, на выходах всех элементов $E, E_{1}, \ldots, E_{r}$ реализуются константы. 
Значит, все эти элементы - конъюнкторы и на их выходах реализуются константы 0. Неисправность типа „константа 0“ на выходе элемента $E^{\prime}$ нетривиальная, но обнаружить ее нельзя, так как при этой неисправности на выходах $E, E_{1}, \ldots, E_{r}$ снова окажется реализованной константа 0. Теорема доказана.

Следствие 1. Для каждой равной константе булевой функиии $f$ не существует реализующей ее неизбыточной СФЭ с одним выходом в базисах $B_{3}=\{x \mid y\}, B_{4}=$ $\{x \downarrow y\}$.

Доказательство вытекает из теоремы 4 , лемм $3-5$ и того, что $\bar{x}=x \mid x=x \downarrow x$.

\section{Синтез легкотестируемых неизбыточных схем в бази- $\operatorname{cax} B_{5}=\{\bar{x}, x \& \bar{y}\}, B_{6}=\{\bar{x}, x \vee \bar{y}\}$}

Теорема 6. Для каждой булевой функиии $f$ существует реализующая ее неизбыточная $С Ф \ni S_{B}^{f}$ в базисе $B \in\left\{B_{5}, B_{6}\right\}$ (где $B_{5}=\{\bar{x}, x \& \bar{y}\}, B_{6}=\{\bar{x}, x \vee \bar{y}$, допускающая единичный проверяющий тест длинь, не превосходящей четырех, относительно инверсных и произвольных константных неисправностей на выходах элементов.

Доказательство. Рассмотрим базис $B_{5}$. Пусть, как и ранее, $g(x, y)=x \& \bar{y}$. Для функции $f$, равной нулю, единице, $x_{1}, \bar{x}_{1}$ искомые схемы моделируют формулы $g(x, x), \bar{g}(x, x), x_{1}$ или $\bar{x}_{1}$ соответственно. Пусть теперь функция $f$ существенно зависит не менее чем от двух переменных. Воспользуемся схемой $S_{B^{\prime \prime}}^{f}$, построенной в теореме 2 . Пусть $E$ - какой-то элемент суммы по модулю 2 или эквивалентности в этой схеме. Пусть на входных наборах из $\check{T}_{n}$ (из теоремы 2) на входах этого элемента возникает тройка различных наборов $A$ из множества $\{(0,0),(0,1),(1,0)$, $(1,1)\}$ (такая тройка найдется по части 2 теоремы 2) и на каждом наборе из $A$ будет обнаруживаться какая-то константная неисправность на выходе $E$. Тогда по лемме 13 найдется эквивалентная элементу $E$ неизбыточная схема $S$ в базисе $B_{5}$, для которой множество $A$ будет единичным проверяющим тестом. Осуществим эквивалентную подстановку схемы $S$ вместо элемента $E$. Выполним аналогичную процедуру для каждого элемента суммы по модулю 2 или эквивалентности в схеме $S_{B^{\prime \prime}}^{f}$. По лемме 2 полученная схема $S_{B_{5}}^{f}$ в базисе $B_{5}$ неизбыточна, а множество $\check{T}_{n}$ образует для нее единичный проверяющий тест.

Чтобы доказать теорему для базиса $B_{6}$, надо воспользоваться принципом двойственности. Теорема доказана.

\section{Синтез легкотестируемых неизбыточных схем в про- извольном базисе $B$}

Расширением базиса $B$ называется такой базис, что каждый его элемент либо содержится в $B$, либо получается из какого-то элемента $B$ отождествлением его входов. Максимальное по включению элементов расширение базиса $B$ называется максимальным расширением и обозначается через $\check{B}$.

В дальнейшем, если элемент есть в максимальном расширении базиса, то будем считать, что он есть и в самом базисе. 
Лемма 16. Пусть расширение $\check{B}$ полного базиса $B$ содержит обе константы 0 и 1. Тогда в этом базисе можно построить неизбыточную схему $\tilde{S}_{B}^{\bar{x}}$, реализуюшую функиию $\bar{x}$.

Доказательство. В силу полноты в базисе $B$ есть немонотонная функция $f_{M}\left(x_{1}, \ldots, x_{t}\right)$. Пусть $\tilde{\alpha}=\left(\alpha_{1}, \ldots, \alpha_{t}\right)$ и $\tilde{\beta}=\left(\beta_{1}, \ldots, \beta_{t}\right)$ - наиболее удаленные друг от друга (в смысле расстояния Хэмминга) наборы, на которых нарушается монотонность $f_{M}$. Не ограничивая общности, можно считать, что

$$
\begin{gathered}
\tilde{\alpha}=(\underbrace{0, \ldots, 0}_{k}, \underbrace{0, \ldots, 0}_{l}, \underbrace{1, \ldots, 1}_{m}), \quad \tilde{\beta}=(\underbrace{0, \ldots, 0}_{k}, \underbrace{1, \ldots, 1}_{l}, \underbrace{1, \ldots, 1}_{m}), \\
f_{M}(\tilde{\alpha})=1, \quad f_{M}(\tilde{\beta})=0, \quad k \geqslant 0, \quad l \geqslant 1, \quad m \geqslant 0 .
\end{gathered}
$$

Построим схему для $\bar{x}$ следующим образом: на первые $k$ входов элемента $f_{M}$ подадим выход элемента „константа $0 “$, на следующие $l$ входов элемента $f_{M}$ подадим вход $x$, на следующие $m$ входов элемента $f_{M}$ подадим выход элемента „константа 1 “. Полученная схема, очевидно, реализует $\bar{x}$. Докажем ее неизбыточность.

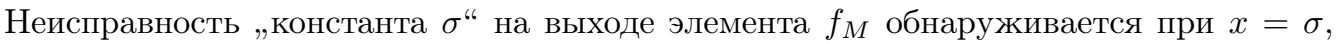
неисправность „константа 1“ на выходе элемента „константа 0“ (если он есть в схеме) обнаруживается при $x=1$, так как в силу максимальной удаленности $\tilde{\alpha}$ и $\tilde{\beta}$ имеем:

$$
f_{M}(\underbrace{1, \ldots, 1}_{k}, \underbrace{1, \ldots, 1}_{l}, \underbrace{1, \ldots, 1}_{m})=1 .
$$

Аналогично, неисправность „константа 0“ на выходе элемента „константа 1“ (если он есть в схеме) обнаруживается при $x=0$. Лемма доказана.

Докажем основную теорему.

Теорема 7. Для каждой отличной от константы булевой функции $f$ существу-

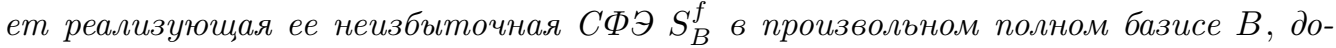
пускающая единичный проверяющий тест длины, не превосходящей четырех, относительно инверсных и произволъных константных неисправностей на выходах элементов.

Доказательство. Заметим, что в силу полноты базиса $B$ в его максимальном расширении есть либо $\bar{x}$, либо обе константы. По лемме 7 в последнем случае можно построить неизбыточную схему для $\bar{x}$. Так как для реализации функции $x$ можно использовать СФЭ без функциональных элементов, то в дальнейшем будем предполагать, что функция $f$ существенно зависит не менее чем от 2 переменных.

В работе Н. П. Редькина [3] (§10) установлен следующий факт: в максимальном расширении $\check{B}$ произвольного полного базиса $B$ содержится или нелинейная функция двух переменных (т. е. одна из функций $x \& y, x \vee y, \bar{x} \& y, \bar{x} \vee y, x \mid y, x \downarrow y$ ), или функция вида $x y \oplus x z \oplus y z \oplus a_{1} x \oplus a_{2} y \oplus a_{3} z \oplus a_{0}\left(a_{i} \in\{0,1\}, i=\overline{0 ; 3}\right)$. Кроме того, там же [3; стр. 213] установлено, что если в максимальном расширении $\check{B}$ произвольного полного базиса $B$ отсутствуют константы, то в нем присутствует хотя бы одна из функций $x \& y, x \vee y, x \mid y, x \downarrow y$.

Разберем соответствующие случаи.

1. В расширении $\check{B}$ содержится функция $x \& y$ (случай функции $x \vee y-$ двойственный). Если в $\check{B}$ содержится также функция $\bar{x}$, то утверждение теоремы следует из теоремы 3 . Если же в $\check{B}$ нет функции $\bar{x}$, то в силу полноты $B$ в $\check{B}$ содержатся обе 
константы. Тогда по лемме 16 в этом базисе можно построить неизбыточную схему $\tilde{S}_{B}^{\bar{x}}$, реализующую функцию $\bar{x}$, заменив на которую все инверторы в неизбыточной схеме $S_{B_{1}}^{f}$ (см. теорему 3 ), получим по лемме 2 неизбыточную схему $S_{B}^{f}$ с тестом длины, не превосходящей четырех.

2. В расширении $\check{B}$ содержится одна из функций $x \mid y, x \downarrow y$. Требуемое утверждение вытекает из теоремы 4.

3. В расширении $\check{B}$ содержится одна из функций $\bar{x} \& y, \bar{x} \vee y$. Этот случай полностью аналогичен случаю $\mathbf{1}$, но требуется ссылаться на теорему 6 вместо теоремы 3 .

4. В расширении $\check{B}$ содержится функция $\varkappa_{1}(x, y, z)=x y \oplus x z \oplus y z \oplus x \oplus y \oplus z \oplus 1$ (и, следовательно, константа 1). Вектор-столбец функции $\varkappa_{1}(x, y, z)$ имеет вид (10000001). Построим схему $\tilde{S}_{B}^{(1)}$, подав на вход $z$ элемента $e_{\varkappa_{1}}$, реализующего $\varkappa_{1}(x, y, z)$, выход элемента „константа 1“. Эта схема реализует функцию $x \& y$, неизбыточна и при этом, как это следует из вектора значений функции $\varkappa_{1}(x, y, z)$ и из способа построения схемы $\tilde{S}_{B}^{(1)}$, набор $(0,0)$ обнаруживает константную неисправность типа 1 элемента $e_{\varkappa_{1}}$ и константную неисправность типа 0 элемента „константа 1 “, каждый из наборов $(0,1),(1,0)$ обнаруживает только константную неисправность типа 1 элемента $e_{\varkappa_{1}}$, набор $(1,1)$ обнаруживает константную неисправность типа 0 элемента $e_{\varkappa_{1}}$ и константную неисправность типа 0 элемента „константа $1 “$. Из случая 1 доказательства следует существование неизбыточной схемы $S_{\tilde{B}}^{f}$ для базиса $\tilde{B}=B \cup\{x \& y\}$, обладающей единичным проверяющим тестом $\hat{T}_{n}$ длины не более 4 . Заменим все конъюнкторы в схеме $S_{\tilde{B}}^{f}$ на эквивалентные им подсхемы $\tilde{S}_{B}^{(1)}$ - получим схему $\hat{S}_{B}^{f}$. Так как схема $S_{\tilde{B}}^{f}$ неизбыточна и обладает тестом не более чем из 4 наборов, то для каждого ее конъюнктора $E_{\&}$ найдутся два набора из теста, обнаруживающих различные константные неисправности на выходе $E_{\&}$ и порождающие два набора на входах конъюнктора, один из которых $(1,1)$. Это означает, что получившаяся схема $\hat{S}_{B}^{f}$ неизбыточна, и все нетривиальные константные неисправности на выходах ее элементов обнаруживаются на наборах из $\hat{T}_{n}$.

5. В расширении $\check{B}$ содержится функция $\varkappa_{2}(x, y, z)=x y \oplus x z \oplus y z \oplus x \oplus y \oplus z($, следовательно, константа 0). Этот случай двойственен предыдущему.

6. В расширении $\check{B}$ содержится функция $\varkappa_{3}(x, y, z)=x y \oplus x z \oplus y z \oplus x \oplus y \oplus 1$ и константа $b$. Вектор-столбец функциии $\varkappa_{3}(x, y, z)$ имеет вид (11010100). Построим схему $\tilde{S}_{B}^{(3)}$, подав на вход $z$ элемента $e_{\varkappa_{3}}$, реализующего $\varkappa_{3}(x, y, z)$, выход элемента „константа $b “$. Эта схема в зависимости от $b$ реализует функцию $x \mid y$ (при $b=1)$ или $x \downarrow y($ при $b=0$ ), неизбыточна и при этом набор $(b, b)$ обнаруживает только константную неисправность типа $b$ элемента $e_{\varkappa_{3}}$, каждый из наборов $(0,1)$, $(1,0)$ обнаруживает константную неисправность типа $\bar{b}$ элемента $e_{\varkappa_{3}}$ и константную неисправность типа $\bar{b}$ элемента „константа $b^{“}$, набор $(\bar{b}, \bar{b})$ обнаруживает только константную неисправность типа $\bar{b}$ элемента $e_{\varkappa_{3}}$. Из случая 2 доказательства следует существование неизбыточной схемы $S_{\tilde{B}}^{f}$ для базиса $\tilde{B}=B \cup\left\{\varkappa_{3}(x, y, b)\right\}$, обладающей тестом $\hat{T}_{n}$ из не более чем 4 наборов. Заменим все функциональные элементы для $g_{3, b}(x, y)=\varkappa_{3}(x, y, b)$ в схеме $S_{\tilde{B}}^{f}$ на эквивалентные им подсхемы $\tilde{S}_{B}^{(3)}-$ получим схему $\hat{S}_{B}^{f}$. Так как схема $S_{\tilde{B}}^{f}$ неизбыточна и обладает тестом $\hat{T}_{n}$ не более чем из 4 наборов, то для каждого ее элемента $E_{g_{3, b}}$ (которому приписана функция $g_{3, b}(x, y)$ ) найдутся два набора из теста, обнаруживающих различные константные неисправности на выходе $E_{g_{3, b}}$ и порождающие два набора на входах $E_{g_{3, b}}$, один из которых $(b, b)$. Это означает, что в получившейся схеме $\hat{S}_{B}^{f}$ могут не обнаруживаться только неисправности типа $\bar{b}$ некоторых элементов „константа $b^{“}$. Определим понятие 


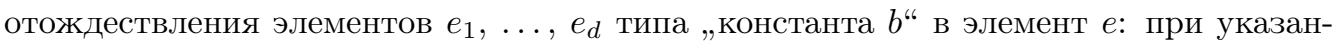
ном отождествлении сами эти элементы удаляются из схемы, добавляется новый элемент $e$ типа „константа $b “$, и выход этого нового элемента подается на те входы элементов, на которые подавались выходы элементов $e_{1}, \ldots, e_{d}$. Отождествим все элементы „константа $b^{“}$, неисправности которых типа „константа $\bar{b}^{“}$ не обнаруживаются на наборах из $\hat{T}_{n}$, в один функциональный элемент $E_{1}$. Если неисправность типа „константа $\bar{b}^{“}$ функционального элемента $E_{1}$ не обнаруживается на наборах из $\hat{T}_{n}$, то добавим еще один элемент $E_{1}^{\prime}$ типа „константа $b^{“}$, подадим выходы $E_{1}$ и $E_{1}^{\prime}$ на два правых входа нового элемента $E_{\varkappa_{3}}$ (типа $\varkappa_{3}$ ), на левый вход элемента $E_{\varkappa_{3}}$ подадим выход схемы $\hat{S}_{B}^{f}$, выход $E_{\varkappa 3}$ подадим на три входа еще одного нового элемента $E_{\varkappa_{3}}^{\prime}\left(\right.$ типа $\left.\varkappa_{3}\right)$ и перенесем выход схемы на выход элемента $E_{\varkappa_{3}}^{\prime}$, - получим схему $S_{B}^{f}$. Из столбца значений функции $\varkappa_{3}(x, y, z)$ и из способа построения схемы ясно, что 1) при отсутствии неисправностей полученная схема реализует функцию $f\left(\right.$ так как $\left.\left.\varkappa_{3}(f, b, b)=\bar{f}, \varkappa_{3}(\bar{f}, \bar{f}, \bar{f})=f\right), 2\right)$ любая неисправность, обнаруживаемая на наборах теста в схеме $\hat{S}_{B}^{f}$, обнаруживается и в схеме $S_{B}^{f}$, и, наконец, 3) неисправности типа $\bar{b}$ элементов $E_{1}, E_{1}^{\prime}$ и константные неисправности элементов $E_{\varkappa_{3}}, E_{\varkappa_{3}}^{\prime}$ обнаруживаются на наборах $\hat{T}_{n}$ в $S_{B}^{f}$ (последнее очевидно, а первое следует из того, что $\left.\varkappa_{3}(f, b, \bar{b})=\bar{b}, \varkappa_{3}(f, \bar{b}, b)=b\right)$.

7. В расширении $\check{B}$ содержатся функция $\varkappa_{4}(x, y, z)=x y \oplus x z \oplus y z \oplus x \oplus y$ и константа $b$. Вектор-столбец функциии $\varkappa_{4}(x, y, z)$ имеет вид (00101011). Построим схему $\tilde{S}_{B}^{(4)}$, подав на вход $z$ элемента $e_{\varkappa_{4}}$, реализующего $\varkappa_{4}(x, y, z)$, выход элемента „константа $b^{“}$. Эта схема в зависимости от $b$ реализует функцию $x \& y$ (при $b=1)$ или $x \vee y($ при $b=0)$, неизбыточна и при этом набор $(b, b)$ обнаруживает только константную неисправность типа $\bar{b}$ элемента $e_{\varkappa_{4}}$, каждый из наборов $(0,1)$, $(1,0)$ обнаруживает константную неисправность типа $b$ элемента $e_{\varkappa_{4}}$ и константную неисправность типа $\bar{b}$ элемента „константа $b^{“}$, набор $(\bar{b}, \bar{b})$ обнаруживает только константную неисправность типа $b$ элемента $e_{\varkappa_{4}}$. Из случая 2 доказательства следует существование неизбыточной схемы $S_{\tilde{B}}^{f}$ для базиса $\tilde{B}=B \cup\left\{\varkappa_{4}(x, y, b)\right\}$, обладающей тестом из не более чем 4 наборов. Заменим все функциональные элементы для $g_{4, b}(x, y)=\varkappa_{4}(x, y, b)$ в схеме $S_{\tilde{B}}^{f}$ на эквивалентные им подсхемы $\tilde{S}_{B}^{(4)}-$ получим схему $\hat{S}_{B}^{f}$. Так как схема $S_{\tilde{B}}^{f}$ неизбыточна и обладает тестом $\hat{T}_{n}$ не более чем из 4 наборов, то для каждого ее элемента $E_{g_{4, b}}$ (которому приписана функция $g_{4, b}$ ) найдутся два набора из теста, обнаруживающих различные константные неисправности на выходе $E_{g_{4, b}}$ и порождающие два набора на входах $E_{g_{4, b}}$, один из которых $(b, b)$. Это означает, что в получившейся схеме $\hat{S}_{B}^{f}$ могут не обнаруживаться только неисправности типа $\bar{b}$ некоторых элементов „константа $b^{“}$. Отождествим все элементы типа „константа $b^{“}$, чьи неисправности типа „константа $\bar{b}^{“}$ не обнаруживаются на наборах из $\hat{T}_{n}$, в один функциональный элемент $E_{1}$. Если неисправность типа

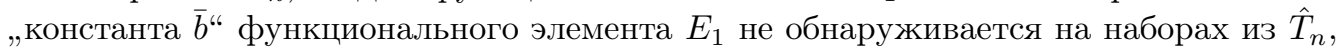
то добавим еще один элемент $E_{1}^{\prime}$ типа „константа $b^{“}$, подадим выходы $E_{1}$ и $E_{1}^{\prime}$ на два правых входа нового элемента $E_{\varkappa_{4}}$ (ему приписана функция $\left.\varkappa_{4}\right)$, на левый вход элемента $E_{\varkappa_{4}}$ подадим выход схемы $\hat{S}_{B}^{f}$ и перенесем выход схемы на выход элемента $E_{\varkappa_{4}}$, - получим схему $S_{B}^{f}$. Из столбца значений функции $\varkappa_{4}(x, y, z)$ и из способа построения схемы ясно, что 1) при отсутствии неисправностей полученная схема реализует функцию $f$ (так как $\left.\varkappa_{4}(f, b, b)=f\right), 2$ ) любая неисправность, обнаруживаемая на наборах теста в схеме $\hat{S}_{B}^{f}$, обнаруживается и в схеме $S_{B}^{f}$, и, наконец, 3$)$ неисправности типа $\bar{b}$ элементов $E_{1}, E_{1}^{\prime}$ и константные неисправности элемента $E_{\varkappa_{4}}$ 
обнаруживаются на наборах $\hat{T}_{n}$ в $S_{B}^{f}$ (последнее очевидно, а первое следует из того, что $\left.\varkappa_{4}(f, b, \bar{b})=b, \varkappa_{4}(f, \bar{b}, b)=\bar{b}\right)$.

8. В расширении $\check{B}$ содержится функция $\varkappa_{5}(x, y, z)=x y \oplus x z \oplus y z \oplus z \oplus 1$ (и, следовательно, константа 1). Вектор-столбец функции $\varkappa_{5}(x, y, z)$ имеет вид (10111101). Построим схему $\tilde{S}_{B}^{(5)}$, подав на вход $z$ элемента $e_{\varkappa_{5}}$, реализующего $\varkappa_{5}(x, y, z)$, выход элемента „константа 1“. Эта схема реализует функцию $x \vee y$, неизбыточна и при этом единичным проверяющим тестом для нее является любой тест из двух наборов, среди которых есть $(0,0)$. Из случая 1 доказательства следует существование неизбыточной схемы $S_{\tilde{B}}^{f}$ для базиса $\tilde{B}=B \cup\{x \vee y\}$, обладающей тестом из не более чем 4 наборов. Заменим все дизъюнкторы в схеме $S_{\tilde{B}}^{f}$ на эквивалентные им подсхемы $\tilde{S}_{B}^{(5)}-$ получим схему $S_{B}^{f}$. Так как схема $S_{\tilde{B}}^{f}$ неизбыточна и обладает тестом не более чем из 4 наборов, то для каждого ее дизъюнктора $E_{\vee}$ найдутся два набора из теста, обнаруживающих различные константные неисправности на выходе $E_{\vee}$ и порождающие два набора на входах дизъюнктора, один из которых $(0,0)$. Это означает, что получившаяся схема $S_{B}^{f}$ неизбыточна и обладает тестом не более чем из 4 наборов.

9. В расширении $\check{B}$ содержится функция $\varkappa_{6}(x, y, z)=x y \oplus x z \oplus y z \oplus z$ (и, следовательно, константа 0). Этот случай двойствен предыдущему.

10. В расширении $\check{B}$ содержится функция $\varkappa_{7}(x, y, z)=x y \oplus x z \oplus y z \oplus 1$ и константа $b$. Построим схему $\tilde{S}_{B}^{(7)}$, подав на вход $z$ элемента $e_{\varkappa_{7}}$, реализующего $\varkappa_{7}(x, y, z)$, выход элемента „константа $b$ “. Эта схема в зависимости от $b$ реализует функцию $x \mid y$ $($ при $b=0)$ или $x \downarrow y($ при $b=1)$, неизбыточна и при этом набор $(\bar{b}, \bar{b})$ обнаруживает только константную неисправность типа $\bar{b}$ элемента $e_{\varkappa_{7}}$, каждый из наборов $(0,1)$, $(1,0)$ обнаруживает константную неисправность типа $b$ элемента $e_{\varkappa 7}$ и константную неисправность типа $\bar{b}$ элемента „константа $b^{“}$, набор $(b, b)$ обнаруживает только константную неисправность типа $b$ элемента $e_{\varkappa 7}$. Из случая 2 доказательства следует существование неизбыточной схемы $S_{\tilde{B}}^{f}$ для базиса $\tilde{B}=B \cup\left\{\varkappa_{7}(x, y, b)\right\}$, обладающей единичным проверяющим тестом $\hat{T}_{n}$ из не более чем 4 наборов. Заменим все функциональные элементы для $g_{7, b}(x, y)=\varkappa_{7}(x, y, b)$ в схеме $S_{\tilde{B}}^{f}$ на эквивалентные им подсхемы $\tilde{S}_{B}^{(7)}-$ получим схему $\hat{S}_{B}^{f}$. Так как схема $S_{\tilde{B}}^{f}$ неизбыточна и обладает тестом не более чем из 4 наборов, то для каждого ее элемента $E_{g_{7, b}}$ (типа $\left.g_{7, b}(x, y)\right)$ найдутся два набора из теста, обнаруживающих различные константные неисправности на выходе $E_{g_{7, b}}$ и порождающие два набора на входах $E_{g_{7, b}}$, один из которых $(\bar{b}, \bar{b})$. Это означает, что в получившейся схеме $\hat{S}_{B}^{f}$ могут не обнаруживаться только неисправности типа $\bar{b}$ некоторых элементов „константа $b^{“}$. Отождествим все элементы типа „константа $b^{“}$, неисправности которых типа „константа $\bar{b}^{“}$ не обнаруживаются на наборах из $\hat{T}_{n}$, в один функциональный элемент $E_{1}$. Если неисправность типа „константа $\bar{b}^{“}$ функционального элемента $E_{1}$ не обнаруживается на наборах из $\hat{T}_{n}$, то добавим еще один элемент $E_{1}^{\prime}$ типа „константа $b^{“}$, подадим выход $E_{1}$ на три входа нового элемента $E_{\varkappa_{7}}$ (типа $\varkappa_{7}$ ), выход $E_{\varkappa_{7}}$ и выход $E_{1}^{\prime}$ подадим на два левых входа нового элемента $E_{\varkappa_{7}}^{\prime}$ (типа $\varkappa_{7}$ ), на третий вход элемента $E_{\varkappa_{7}}^{\prime}$ подадим выход схемы $\hat{S}_{B}^{f}$, выход $E_{\varkappa_{7}}^{\prime}$ подадим на три входа еще одного нового элемента $E_{\varkappa_{7}}^{\prime \prime}\left(\right.$ типа $\left.\varkappa_{7}\right)$ и перенесем выход схемы на выход элемента $E_{\varkappa_{7}}^{\prime \prime}$, - получим схему $S_{B}^{f}$. Из столбца значений функции $\varkappa_{7}$ и способа построения схемы вытекает, что 1) при отсутствии неисправностей полученная схема реализует функцию $f$ (так как $\left.\left.\varkappa_{7}(b, \bar{b}, f)=\bar{f}, \varkappa_{7}(\bar{f}, \bar{f}, \bar{f})=f\right), 2\right)$ любая неисправность, обнаруживаемая на на- 
борах теста в схеме $\hat{S}_{B}^{f}$, обнаруживается и в схеме $S_{B}^{f}$, и, наконец, 3 ) неисправности типа $\bar{b}$ элементов $E_{1}, E_{1}^{\prime}$ и нетривиальные константные неисправности элементов $E_{\varkappa_{7}}, E_{\varkappa_{7}}^{\prime}, E_{\varkappa_{7}}^{\prime \prime}$ обнаруживаются на наборах $\hat{T}_{n}$ в $S_{B}^{f}$ (последнее несложно, а первое следует из того, что $\left.\varkappa_{7}(b, b, f)=\bar{b}, \varkappa_{7}(\bar{b}, \bar{b}, f)=b\right)$.

11. В расширении $\check{B}$ содержатся функция $\varkappa_{8}(x, y, z)=x y \oplus x z \oplus y z$ и константа $b$. Построим схему $\tilde{S}_{B}^{(8)}$, подав на вход $z$ элемента $e_{\varkappa_{8}}$, реализующего $\varkappa_{8}(x, y, z)$, выход элемента „константа $b$ “. Эта схема в зависимости от $b$ реализует функцию $x \& y$ $($ при $b=0)$ или $x \vee y($ при $b=1)$, неизбыточна и при этом набор $(b, b)$ обнаруживает только константную неисправность типа $\bar{b}$ элемента $e_{\varkappa 8}$, каждый из наборов $(0,1)$, $(1,0)$ обнаруживает константную неисправность типа $\bar{b}$ элемента $e_{\varkappa_{8}}$ и константную неисправность типа $\bar{b}$ элемента „константа $b^{“}$, набор $(\bar{b}, \bar{b})$ обнаруживает только константную неисправность типа $b$ элемента $e_{\varkappa_{8}}$. Из случая 1 доказательства вытекает существование неизбыточной схемы $S_{\tilde{B}}^{f}$ для базиса $\tilde{B}=B \cup\{\varkappa(x, y, b)\}$, обладающей единичным проверяющим тестом $\hat{T}_{n}$, состоящим из не более чем 4 наборов. Заменим все функциональные элементы для $g(x, y)=\varkappa_{8}(x, y, b)$ в схеме $S_{\tilde{B}}^{f}$ на эквивалентные им подсхемы $\tilde{S}_{B}^{(8)}-$ получим схему $\hat{S}_{B}^{f}$. Так как схема $S_{\tilde{B}}^{f}$ неизбыточна и обладает тестом не более чем из 4 наборов, то для каждого ее элемента $E_{g}$ (типа $g(x, y)$ ) найдутся два набора из теста, обнаруживающих различные константные неисправности на выходе $E_{g}$ и порождающие два набора на входах $E_{g}$, один из которых $(b, b)$. Это означает, что в получившейся схеме $\hat{S}_{B}^{f}$ могут не обнаруживаться только неисправности типа $\bar{b}$ некоторых элементов „константа $b^{“}$. Отождествим все элементы типа „константа $b^{“}$, неисправности которых типа „константа $\bar{b}^{“}$ не обнаруживаются на наборах из $\hat{T}_{n}$, в один функциональный элемент $E_{1}$. Если неисправность типа „константа $\bar{b}^{“}$ функционального элемента $E_{1}$ не обнаруживается на наборах из $\hat{T}_{n}$, то добавим еще один новый элемент $E_{\varkappa_{8}}$ (типа $\left.\varkappa_{8}\right)$ и подадим на его левый вход выход $E_{1}$, на правый - выход схемы $\hat{S}_{B}^{f}$. Из полноты базиса $B$ следует, что в $\check{B}$ есть либо обе константы, либо $\bar{x}$. В зависимости от этого добавим еще либо элемент $E_{1}^{\prime}$ типа „константа $\bar{b}^{“}$ и его выход подадим на средний вход $E_{\varkappa_{8}}$, либо элемент $E_{1}^{\prime \prime}$ типа „константа $b^{“}$ и инвертор $E_{-}$, выход $E_{1}^{\prime \prime}$ подадим на $E_{-}$, а выход $E_{-}$подадим на средний вход $E_{\varkappa 8}$, - получим схему $S_{B}^{f}$. Ясно, что 1) при отсутствии неисправностей полученная схема реализует функцию $f\left(\right.$ так как $\left.\varkappa_{8}(b, \bar{b}, f)=f\right), 2$ ) любая неисправность, обнаруживаемая на наборах теста в схеме $\hat{S}_{B}^{f}$, обнаруживается и в схеме $S_{B}^{f}$, и, наконец, 3) нетривиальные неисправности добавленных элементов обнаруживаются на наборах $\hat{T}_{n}$ в $S_{B}^{f}\left(\right.$ так как $\left.\varkappa_{8}(b, b, f)=b, \varkappa_{8}(\bar{b}, \bar{b}, f)=\bar{b}\right)$.

Теорема доказана.

Автор выражает глубокую благодарность профессору Сергею Андреевичу Ложкину за обсуждение работы и ценные замечания, а также рецензенту статьи за значительные усилия, приведшие к улучшению текста.

\section{Список литературы}

1. Яблонский С. В., Чегис И. А., "О тестах для электрических схем", Успехи матем. наук, 10:4 (66) (1955), 182-184.

2. Чегис И. А., Яблонский С. В., "Логические способы контроля электрических схем", Труды МИ АН СССР, 51 (1958), 270-360.

3. Редькин Н. П., Надежность и диагностика схем, М.: Изд-во МГУ, 1992.

5 Дискретная математика, т. 26 № 2 
4. Reddy S. M., "Easily testable realization for logic functions", IEEE Trans. Comput., 21:1 (1972), 124-141.

5. Редькин Н. П., "О схемах, допускающих короткие тесты”, Вестник Моск. ун-та. Серия 1. Матем. Механика., 1988, № 2, 17-21.

6. Редькин Н. П., "О полных проверяющих тестах для схем из функциональных элементов", Матем. вопросы кибернетики, 1989, № 2, 198-222.

7. Коваценко С. В., "Синтез легкотестируемых схем в базисе Жегалкина для инверсных неисправностей”, Вестник Моск. ун-та. Серия 15. Вычислит. матем. и киберн., 2000, № 2, 45-47.

8. Редькин Н. П., "Единичные проверяющие тесты для схем при инверсных неисправностях элементов", Матем. вопросы кибернетики. Вып. 12, 2003, 217-230.

9. Бородина Ю. В., "О синтезе легкотестируемых схем в случае однотипных константных неисправностей на выходах элементов", Вестник Моск. ун-та. Серия 15. Вычислит. матем. и киберн., 2008, № 1, 40-44.

10. Бородина Ю. В., “О схемах, допускающих единичные тесты длины 1 при константных неисправностях на выходах элементов", Вестник Моск. ун-та. Серия 1. Матем. Механика, 5 (2008), 49-52.

11. Бородина Ю. В., Бородин П. А., "Синтез легкотестируемых схем в базисе Жегалкина при константных неисправностях типа „0“ на выходах элементов”, Дискретная математика, 22:3 (2010), 127-133.

12. Коляда С. С., "О единичных проверяющих тестах для константных неисправностей на выходах функциональных элементов", Вестн. Моск. ун-та. Сер. 1. Матем. Механика, 2011, № 6, 47-49.

13. Коляда С. С., "Единичные проверяющие тесты для схем из функциональных элементов в базисах из элементов, имеющих не более двух входов", Дискретн. анализ и исслед. опер., 20:2 (2013), 58-74.

14. Коляда С. С., "Единичные проверяющие тесты для схем из функциональных элементов", Вестн. Моск. ун-та. Сер. 1. Матем. Механик, 2013, № 4, 32-34.

15. Романов Д. С., "Метод синтеза легкотестируемых схем в одном базисе, допускающих единичные проверяющие тесты константной длины”, Вестн. Моск. ун-та. Матем. Механ., 2012, № 2, 24-29.

16. Романов Д. С., "О синтезе схем, допускающих полные проверяющие тесты константной длины относительно произвольных константных неисправностей на выходах элементов", Дискретная математика, 25:2 (2013), 104-120.

17. Носков В. Н., "Метод синтеза удобных для контроля комбинационных схем", Дискретная математика, 5:4 (1993), 3-23. 